\title{
ON THE NON-COMMUTATIVITY OF PONTRJAGIN RINGS MOD 3 OF SOME COMPACT EXCEPTIONAL GROUPS
}

\author{
SHÔRÔ ARAKI
}

Introduction. Pontrjagin rings over the field of rational numbers of compact Lie groups are commutative in the sense of graded algebras (or anticommutative in the classical terminology) [14]. Pontrjagin rings over the field $Z_{p}(p \neq 0)$ of several compact simple Lie groups were studied by Borel [5]. The most examples are commutative. However, this is generally not true.

The first example of non-commutative Pontrjagin rings of compact Lie groups is $H_{*}\left(\operatorname{Spin}(10) ; Z_{2}\right),[5],[1]$. Then it was shown that the Pontrjagin rings $H_{*}\left(\operatorname{Spin}(n) ; Z_{2}\right), n \geqq 10$ and $n \neq 2^{s}+1$, are non-commutative, [11], [18]. These known examples are all those over $Z_{2}$.

In this work we prove that the Pontrjagin rings mod 3 of all compact exceptional groups except $G_{2}$ are not commutative.

We denote by $F_{4}, E_{6}, E_{7}$ and $E_{3}$ the compact, connected and simply-connected groups among the local structures usually expressed by these notations.

In Chapter I we determine the ring structure of the Pontrjagin ring $H_{*}\left(F_{4} ; Z_{3}\right)$. This is shown to be non-commutative. The proof is based on a theorem of Kudo [12] on one hand, and on the scheme used by Borel [5] to determine the Pontrjagin ring $H_{*}\left(\operatorname{Spin}(10) ; Z_{2}\right)$ on the other hand.

In Chapter II we discuss the homology maps $H_{*}\left(\Omega F_{4} ; Z\right) \rightarrow H_{*}\left(\Omega E_{6} ; Z\right)$ in degrees $\leqq 10, H_{*}\left(\Omega E_{6} ; Z\right) \rightarrow H_{*}\left(\Omega E_{7} ; Z\right)$ in degrees $\leqq 10$, and $H_{*}\left(\Omega E_{7} ; Z\right)$ $\rightarrow H_{*}\left(\Omega E_{8} ; Z\right)$ in degrees $\leqq 14$, induced by the inclusions $F_{4} \subset E_{6} \subset E_{7} \subset E_{8}$. In the description of these homology maps we use $K$-cycles due to BottSamelson [10].

In Chapter III we prove that the Pontrjagin rings $\bmod 3$ of $E_{6}, \operatorname{Ad} E_{6}, E_{7}$ and $E_{8}$ are non-commutative using the results of Chapters I and II.

The author is thankful to Professor Armand Borel who attracted my interests in this direction and could give me valuable discussions.

Received June 3, 1960. 


\section{Chapter I. The Pontrjagin ring $\boldsymbol{H}_{*}\left(F_{4} ; Z_{3}\right)$}

\section{$\S 1$. Kudo theorem and the comparison theorem of spectral sequences}

1. Let $\left\{E_{r}, r \geqq 0\right\}$ be the cohomology spectral sequence of Serre [15] over $Z_{p}$ ( $p$ is an odd prime) associated with a fibration. About terminologies and notations of cohomology spectral sequences we refer to [4], [15]. Kudo theorem on transgressions [12] is stated as follows:

Theorem K. Let $\alpha \in E_{2}^{o, 2 k}(k>0)$ be transgressive. Choose a suitable representative $\beta \in E_{2}^{2 k+1, o}$ of $\tau(\alpha)$, then

i) $\alpha^{p}$ and $\beta \cdot \alpha^{p-1}$ are transgressive,

ii) $\mathscr{P}_{p}^{k} \beta$ and $-\delta_{p}^{*} \mathscr{P}_{p}^{k} \beta \in E_{2}^{* o}$, represents $\tau\left(\alpha^{p}\right)$ and $\tau\left(\beta \cdot \alpha^{p-1}\right)$ respectively, where $\tau$ denotes the transgression

$$
d_{b+1} \kappa_{b+1}^{2}:\left(\kappa_{b+1}^{2}\right)^{-1}\left(E_{b+1}^{a, b}\right) \rightarrow E_{b+1}^{a+b+1, o}
$$

for each $(a, b), \mathscr{P}_{p}^{k}$ a cyclic p-th reduced power and $\delta_{p}^{*}$ the Bockstein operation $\bmod p$.

2. Let $\left\{E_{r} ; r \geqq 2\right\}$ and $\left\{{ }^{\prime} E_{r} ; r \geqq 2\right\}$ be canonical cohomology spectral sequences ([4], p. 122) of modules (or of algebras) such that $E_{2}^{a, b} \approx E_{2}^{a, o} \otimes E_{2}^{o, b}$ and $E_{2}^{a, b} \approx{ }^{\prime} E_{2}^{a, o} \otimes{ }^{\prime} E_{2}^{o, b}$ for all $(a, b) \geqq 0$. Let

$$
\left\{h_{r}, r \geqq 2\right\}:\left\{E_{r} ; r \geqq 2\right\} \rightarrow\left\{E_{r} ; r \geqq 2\right\}
$$

be a homomorphism of the spectral sequences such that

$$
h_{2}^{a, b} \approx h_{2}^{a, o} \otimes h_{2}^{o, b} \quad \text { for all }(a, b) \geqq 0 .
$$

The comparison theorem of spectral sequences ([13], p. 110) has three statements. Among them the one we use later is the following:

THEOREM CSS. Let $h_{2}^{o, b}$ be isomorphic for all $b \geqq 0$ and $h_{\infty}^{a, b}$ be isomorphic for all $(a, b) \geqq 0$. Then $h_{2}^{a, o}$ is isomorphic for all $a \geqq 0$.

The conditions and the conclusion of this theorem can be weakened as in the following:

THEOREM WCSS. Let $h_{2}^{o, b}$ be isomorphic for $0 \leqq b \leqq n$ and $h_{\infty}^{a, b}$ be isomorphic for $0 \leqq a+b \leqq n+1$. Then $h_{2}^{a, o}$ is isomorphic for $0 \leqq a \leqq n+1$.

This theorem is proved in the same way with the proof of the Theorem CSS so that the proof is omitted. 


\section{§. Universal cohomology spectral sequence of $F_{4}$ over $Z_{3}$}

1. By [5] we know that

$$
H^{*}\left(F_{4} ; Z_{3}\right)=\Lambda_{3}\left(x_{3}, x_{7}, x_{11}, x_{15}\right) \otimes Z_{3}\left[x_{8}\right] /\left(x_{8}^{3}\right)
$$

with the following relations of reduced powers and Bockstein operations

$$
x_{7}=\mathscr{P}_{3}^{1} x_{3}, \quad x_{15}=\mathscr{P}_{3}^{1} x_{11}, \quad x_{8}=\delta_{3}^{*} x_{7},
$$

where suffixes denote degrees.

Proposition 1. $H^{*}\left(F_{4} ; Z_{3}\right)$ has no system of universally transgressive generators.

Proof. Assume that $H^{*}\left(F_{4} ; Z_{3}\right)$ has a system of universally transgressive generators : $x_{3}, x_{7}, x_{8}, x_{11}, x_{15}$. We may regard the relation (1) as holding for these generators.

Denoting by $\tau$ the transgression in the universal spectral sequence of $F_{4}$ over $Z_{3}$, by the Theorem $\mathrm{K}$ we can choose representatives

$$
H^{i+1}\left(B_{F_{4}} ; Z_{3}\right) \ni y_{i+1} \text { of } \tau\left(x_{i}\right) \quad(i=3,7,8,11,15)
$$

and

$$
H^{26}\left(B_{F_{4}} ; Z_{2}\right) \ni y_{26} \quad \text { of } \quad \tau\left(y_{9} \otimes x_{8}^{2}\right)
$$

satisfying

$$
y_{9}=-\delta_{3}^{*} y_{8} \text { and } y_{26}=-\delta_{3}^{*} \mathscr{P}_{3}^{4} y_{9} .
$$

Construct a graded algebra

$$
B=Z_{3}\left[y_{4}^{\prime}, y_{8}^{\prime}, y_{12}^{\prime}, y_{16}^{\prime}, y_{26}^{\prime}\right] \otimes \Lambda_{3}\left(y_{9}^{\prime}\right)
$$

and a spectral sequence over $Z_{3}$ which starts from

with

$$
' E_{2}=E_{2}^{*, o} \otimes E_{2}^{o, *}
$$

$$
{ }^{\prime} E_{2}^{*, o}=B \text { and } \quad E_{2}^{o, *}=H *\left(F_{4} ; Z_{3}\right) .
$$

Successive terms of this spectral sequence is defined as follows:

$$
\begin{aligned}
& d_{2}=d_{3}=0, \quad ' E_{2}==^{\prime} E_{3}={ }^{\prime} E_{1} ; \\
& d_{4} \kappa_{4}^{2} x_{3}=\kappa_{4}^{2} y_{4}^{\prime}, \quad d_{4} \kappa_{4}^{2} x_{i}=0 \quad \text { for } i>3,
\end{aligned}
$$

whence ' $E_{5}=H\left({ }^{\prime} E_{4}\right)=B_{1} \otimes C_{1}$ where $B_{1}=B / Z_{3}\left[y_{4}^{\prime}\right]$ and $C_{1}=H^{*}\left(F_{4} ; Z_{3}\right) / / \Lambda_{3}\left(x_{3}\right)$ (// denotes a symbol to take away the tensor factor indicated thereafter. For the strict definition of this symbol as a kind of quotient, cf., J. Milnor and J. 
Moore, "On the structure of Hopf algebras", to appear);

$$
\begin{aligned}
& d_{5}=d_{6}=d_{7}=0, \quad E_{5}={ }^{\prime} E_{6}={ }^{\prime} E_{7}={ }^{\prime} E_{8} ; \\
& d_{8} \kappa_{8}^{2} x_{7}=\kappa_{8}^{2} y_{8}^{\prime}, d_{8} \kappa_{8}^{2} x_{i}=0 \quad \text { for } i>7,
\end{aligned}
$$

whence ' $E_{9}=B_{2} \otimes C_{2}$ where $B_{2}=B_{1} / / Z_{3}\left[y_{8}^{\prime}\right]$ and $C_{3}=C_{1} / / \Lambda_{3}\left(x_{7}\right)$;

$$
d_{9} \kappa_{9}^{2} x_{8}=\kappa_{9}^{2} y_{9}^{\prime} \text { and } d_{9} \kappa_{9}^{2} x_{i}=0 \quad \text { for } i>8 \text {, }
$$

whence ' $E_{10}=B_{3} \otimes C_{3} \otimes \Lambda_{3}\left(x_{25}^{\prime}\right)$ where $B_{3}=B_{2} / / \Lambda_{3}\left(y_{9}^{\prime}\right), C_{3}=C_{2 / /} Z_{3}\left[x_{8}\right] /\left(x_{8}^{3}\right)$ and $x_{25}^{\prime}$ $=\kappa_{10}^{2}\left(y_{9}^{\prime} \otimes x_{8}^{2}\right)$;

$$
\begin{aligned}
& d_{10}=d_{11}=0, \quad E_{10}={ }^{\prime} E_{11}={ }^{\prime} E_{12} ; \\
& d_{12} \kappa_{12}^{2} x_{11}=\kappa_{12}^{2} y_{12}^{\prime}, d_{12} \kappa_{12}^{2} x_{15}=0 \text { and } d_{12} \kappa_{12}^{10} x_{25}^{\prime}=0,
\end{aligned}
$$

whence ' $E_{13}=B_{4} \otimes C_{4} \otimes \Lambda_{3}\left(\kappa_{13}^{10} x_{25}^{\prime}\right)$ where $B_{4}=B_{3} / Z_{3}\left[y_{12}^{\prime}\right]$ and $C_{4}=C_{3} / / \Lambda_{3}\left(x_{11}\right)$;

$$
\begin{aligned}
& d_{13}=d_{14}=d_{15}=0, \quad ' E_{13}={ }^{\prime} E_{14}={ }^{\prime} E_{15}={ }^{\prime} E_{16} ; \\
& d_{16} \kappa_{16}^{2} x_{15}=\kappa_{16}^{2} y_{16}^{\prime} \text { and } d_{16} \kappa_{16}^{10} x_{25}^{\prime}=0,
\end{aligned}
$$

whence ' $E_{17}=Z_{3}\left[\kappa_{17}^{2} y_{26}\right] \otimes \Lambda_{3}\left(\kappa_{17}^{10} x_{25}^{\prime}\right)$;

$$
d_{17} \kappa_{17}^{10} x_{25}^{\prime}=\kappa_{17}^{2} y_{26},
$$

whence ' $E_{18} \approx Z_{3}$ (trivial); finally

$$
\begin{aligned}
& d_{r}=0 \text { for } r>17, \\
& ' E_{18}={ }^{\prime} E_{19}=\cdots={ }^{\prime} E_{\infty} \approx Z_{3}(\text { trivial }) .
\end{aligned}
$$

Denoting by $\left\{E_{r}\right\}$ the universal spectral sequence of $F_{4}$ over $Z_{3}$, next we define a homomorphism of spectral sequences

$$
\left\{h_{r}, r \geqq 2\right\}:\left\{' E_{r}, r \geqq 2\right\} \rightarrow\left\{E_{r}, r \geqq 2\right\}
$$

in the following way:

$$
E_{2}=H^{*}\left(B_{F_{4}} ; Z_{3}\right) \otimes H^{*}\left(F_{4} ; Z_{3}\right)
$$

canonically. Then, $h_{2}$ is defined by

$$
h_{2} y_{i}^{\prime}=y_{i} \quad \text { and } \quad h_{2} x_{i}=x_{i} .
$$

The choices of $x_{i}, y_{i}$ and the construction of ' $E_{r}$ allows us the successive definition of $h_{r}$; namely $h_{2}$ commutes with $d_{2}$ and induces the homomorphism $h_{3}$, $h_{3}$ commutes with $d_{3}$ and induces the homomorphism $h_{1}$, and so on.

Now, since $h_{\infty}:{ }^{\prime} E_{\infty} \approx E_{\infty}$ and $h_{2}^{o, *}:{ }^{\prime} E_{2}^{o, *}=E_{2}^{o, *}$ an identity map, by Theorem CSS we can conclude that 


$$
h_{2}^{*, o}: H^{*}\left(B_{F_{4}} ; Z_{3}\right) \approx B .
$$

In particular $y_{26} \neq 0$ and this is the $\delta_{3}^{*}$-image of $-\mathscr{g}^{4} y_{9}$ by (2). Since $B$ contains only one odd degree generator $y_{9}$ and $\operatorname{deg} \mathscr{P}^{4} y_{9}=25$ is odd, we have

$$
-\mathscr{P}^{4} y_{9}=y_{9} a_{16}
$$

with $a_{16} \in H^{16}\left(B F_{4} ; Z_{3}\right)$. Consider $\delta_{3}^{*} a_{16}=b . \quad b$ has odd degree so that $b=y_{9} c$ with $c \in H^{8}\left(B_{F_{4}} ; Z_{3}\right)$ by the same reason as above. Then, applying $\delta_{3}^{*}$ on both sides of (4) we have

$$
y_{25}=\delta_{3}^{*}\left(y_{9} a_{16}\right)=-y_{9} \delta_{3}^{*} a_{16}=-y_{9} y_{9} c=0 .
$$

This contradicts to (3). Therefore $H^{*}\left(F_{4} ; Z_{3}\right)$ has no system of universally transgressive generators.

(q.e.d.)

2. Remark. The same discussion can be applied to many other compact Lie groups $G$ for which $p$-torsion ( $p$ odd prime) exist and the cohomology ring $H^{*}\left(G ; Z_{p}\right)$ is known. For example,

$$
\begin{array}{ll}
H^{*}\left(P U(p) ; Z_{p}\right)(p \neq 2), & H^{*}\left(E_{6} ; Z_{3}\right), \\
H^{*}\left(E_{7} ; Z_{3}\right), & H^{*}\left(E_{8} ; Z_{3}\right), \quad H^{*}\left(E_{8} ; Z_{5}\right)
\end{array}
$$

have no system of universally transgressive generators. These cohomology rings are determined in $[3 ; 5 ; 6]$. Here we mention only that these cohomology rings contain only one (or two in case $H *\left(E_{8} ; Z_{3}\right)$ ) generator of even degree with height $p$.

But this property does not imply immediately that the "Pontrjagin ring $H_{*}\left(G ; Z_{p}\right)$ is not commutative", as examples of Kojima [11] $H_{*}\left(\operatorname{Spin}\left(2^{s}+1\right)\right.$; $\left.Z_{2}\right)(s \geqq 4)$ show it.

3. By (1) and the Prop. 1 we can determine the behaviors of generators of $H^{*}\left(F_{4} ; Z_{3}\right)$ in the universal spectral sequence.

LEMma 1. $H^{*}\left(B_{F_{4}} ; Z_{3}\right)=Z_{3}\left[y_{4}, y_{8}\right] \otimes A_{3}\left(y_{9}\right)$ in deg. $\leqq 11$ with relations $y_{4}=\tau\left(x_{3}\right), y_{3}=\mathscr{P}^{1} y_{4}$ and $y_{9}=\delta_{3}^{*} y_{s}$.

Proof. By (1) we see immediately that $x_{3}, x_{7}, x_{3}$ are universally transgressive because $x_{3}$ is a generator with the lowest degree and $\mathscr{y}^{1}$ and $\delta_{3}^{*} \mathrm{com}$ mutes with the transgression. Then a similar construction with that in the proof of the Prop. 1 and Theorem WCSS prove this lemma.

(q.e.d.) 
PROPOSITION 2. In the universal spectral sequence $\left\{E_{r}\right\}$ of $F_{4}$ over $Z_{3}$ the behaviors of generators of $H^{*}\left(F_{4} ; Z_{3}\right)$ are as follows: $x_{3}, x_{7}, x_{3}$ are universally transgressive; $x_{11}$ and $x_{15}$ cannot be chosen to be universally transgressive and they can be chosen such that

$$
\begin{aligned}
& d_{i} \kappa_{i}^{2} x_{11}=d_{i} \kappa_{i}^{2} x_{15}=0 \quad \text { for } 2 \leqq i<9, \\
& d_{9} \kappa_{9}^{2} x_{11}=\kappa_{9}^{2}\left(y_{9} \otimes x_{3}\right) \neq 0, \\
& d_{9} \kappa_{9}^{2} x_{15}=\kappa_{9}^{2}\left(y_{9} \otimes x_{7}\right) \neq 0 .
\end{aligned}
$$

Proof. By adding some decomposable elements we can change the generator $x_{11}$ to satisfy

$$
d_{i} \kappa_{i}^{2} x_{11}=0 \quad \text { for } 2 \leqq i \leqq 8 .
$$

If $d_{3} \kappa_{9}^{2} x_{11}=0$, then the generators $x_{11}$ and $x_{15}=\mathscr{P}^{1} x_{11}$ must be universally transgressive. This contradicts to the Prop. 1. Therefore

$$
E_{9}^{9,3} \ni d_{9} \kappa_{9}^{2} x_{11} \neq 0 .
$$

On the other hand $E_{2}^{9,3}$ is 1 -dimensional and generated by $y_{9} \otimes x_{3}$. Hence $y_{9} \otimes x_{3}$ is $d_{r}$-cocycles for all $r \geqq 2, y_{4} y_{9}=0$ and

$$
d_{9} \kappa_{9}^{2} x_{11}=\kappa_{9}^{2}\left(y_{9} \otimes x_{3}\right)
$$

after changing the coefficient of $x_{11}$ suitably. Then $x_{15}=\mathscr{P}^{1} x_{11}$ (the changed generator of deg. 15) is $d_{r}$-cocycles for $2 \leqq r \leqq 8$ and

$$
d_{9} \kappa_{9}^{2} x_{15}=\kappa_{9}^{2}\left(y_{9} \otimes \mathscr{P}^{1} x_{3}\right)=\kappa_{9}^{2}\left(y_{9} \otimes x_{7}\right)
$$

by some properties of reduced powers in spectral sequences [2] (i e., relations with $d_{r}$ and the Cartan formula). This implies that $y_{8} y_{9}=0$. Then $d_{9} \kappa_{9}^{2} x_{15} \neq 0$.

(q.e.d.)

By this proposition we can discuss the universal spectral sequence of $F_{1}$ over $Z_{3}$ in low degrees immediately, and we have

Corollary. $H^{*}\left(B_{F_{4}} ; Z_{3}\right)=Z_{3}\left[y_{4}, y_{8}\right] \otimes \Lambda_{3}\left(y_{9}\right) /\left(y_{1} y_{9}, y_{8} y_{9}\right)$ in deg. $\leqq 19$.

$\S 3$. The coproduct in $H^{*}\left(F_{4} ; Z_{3}\right)$ and the Pontrjagin ring $H_{*}\left(F_{4} ; Z_{3}\right)$

1. For any connected compact Lie group $G$ the group multiplication $h: G \times G$ $\rightarrow G$ induces the coproduct

$$
h^{*}: H^{*}\left(G ; Z_{p}\right) \rightarrow H^{*}\left(G ; Z_{p}\right) \otimes H^{*}\left(G ; Z_{p}\right)
$$

for any prime $p$. For a principal $G$-bundle $(E, B, \pi, G)$ the right translation 
$h: E \times G \rightarrow E$ induces a homomorphism

$$
h^{*}: E_{r} \rightarrow E_{r} \otimes H^{*}\left(G ; Z_{p}\right) \quad(r \geqq 2)
$$

of associated spectral sequences over $Z_{p}[4]$, p. 174. The operation of $u \in H_{s}\left(G ; Z_{p}\right)$ on $H^{*}\left(G ; Z_{p}\right)$ of $\operatorname{deg}-s$ or on $E_{r}$ of $\operatorname{deg}(0,-s)$,

$$
\vartheta_{u} x=\sum<y_{i}, u>x_{i}
$$

where $x \in H^{*}\left(G ; Z_{p}\right)$ or $\in E_{r}$ and $h^{*} x=\sum x_{i} \otimes y_{i}$, was first defined by Leray and then used by Borel [5] for the study of the coproduct in $H *\left(\operatorname{Spin}(10) ; Z_{2}\right)$.

$\vartheta_{u}$ in the spectral sequence of a principal $G$-bundle has the following properties [5]:

i) $\vartheta_{u}$ commutes with $d_{r}$ and $\kappa_{t}^{r}$,

ii) in $E_{2}=H^{*}\left(B ; Z_{p}\right) \otimes H^{*}\left(G ; Z_{p}\right)$ we have $\vartheta_{u}(b \otimes x)=b \otimes \vartheta_{u} x$ for $b \in H^{*}\left(B ; Z_{p}\right)$ and $x \in H^{*}\left(G ; Z_{p}\right)$.

2. THEOREM 1. For generators of $H^{*}\left(F_{4} ; Z_{3}\right)$ which behave in the universal spectral sequence as stated in the Prop. 2, their coproducts are as follows:

a) $x_{3}, x_{7}, x_{8}$ are primitive,

b) $h^{*} x_{11}=1 \otimes x_{11}+x_{11} \otimes 1+x_{3} \otimes x_{3}$,

c) $h^{*} x_{15}=1 \otimes x_{15}+x_{15} \otimes 1+x_{8} \otimes x_{i}$.

Proof. a) is immediate by the Prop. 20.1 of [4].

Let $u_{i}$ be the dual class of $x_{i}$ and $\vartheta_{i}$ be the operation associated with $u_{i}$ $(i=3,8)$. Then

$$
h * x_{11}=1 \otimes x_{11}+x_{11} \otimes 1+\vartheta_{3} x_{11} \otimes x_{3}+\vartheta_{8} x_{11} \otimes x_{8} .
$$

By the Prop. 2 and the property i) of $\vartheta_{u}$ we see that $\vartheta_{8} x_{11}$ is a permanent element. Therefore

$$
\vartheta_{8} x_{11}=0
$$

since universal spectral sequences have no permanent elements except zero. By the Prop. 2 and the properties i) and ii) of $\vartheta_{u}$ we see that $\vartheta_{3} x_{11}$ is $d_{r^{-}}$ cocycles for $2 \leqq r \leqq 8$, and

$$
d_{9} \kappa_{9}^{2} \vartheta_{3} x_{11}=\kappa_{9}^{2} y_{9}=d_{9} \kappa_{9}^{2} x_{8} .
$$

Therefore $\vartheta_{3} x_{11}-x_{8}$ is permanent. Hence

$$
\vartheta_{3} x_{11}=x_{8} .
$$


And $b$ ) is proved.

c) is obtained by applying $\mathscr{D}^{1}$ on both sides of b) since $\mathscr{D}^{1} x_{11}=x_{15}, \mathscr{F}^{1} x_{3}$ $=x_{7}$ and $\mathscr{P}^{1} x_{3}=0$.

(q.e.d.)

3. Since the generators $x_{i}, i=3,7,8,11,15$, form a 3 -simple system of generators of $H^{*}\left(F_{4} ; Z_{3}\right)$ by a terminology of [5],

$$
x_{i_{1}} \cdots x_{i_{r}}, \quad x_{i_{1}} \cdots x_{i_{r}} x_{8}, \quad x_{i_{1}} \cdots x_{i_{r}} x_{8}^{2}
$$

form an additive basis of $H^{*}\left(F_{4} ; Z_{3}\right)$ where $\left\{i_{1}, \ldots, i_{r}\right\}$ are subsequences of $\{3,7,11,15\}$. We denote by $v_{i_{1}, \ldots, i_{r}}, v_{i_{1}}, \ldots, i_{r} ; 1, v_{i_{1}, \ldots, i_{r} ; 2}$ the dual basis in homology. We use the symbol $\left[v_{i}, v_{j}\right]$ to denote $v_{i} \vee v_{j}-(-1)^{i j} v_{j} \vee v_{i}$.

Theorem 2. The Pontrjagin ring $H_{*}\left(F_{4} ; Z_{3}\right)$ is non-commutative and has a 3-simple system of generators $v_{3}, v_{7}, v_{8}, v_{11}, v_{15}$ satisfying the following relations:

$$
\begin{aligned}
& v_{i} \vee v_{i}=0 \text { for } i \neq 8, \quad v_{8} \vee v_{3} \vee v_{8}=0 \\
& {\left[v_{i}, v_{j}\right]=0 \text { for } i<j \text { and }(i, j) \neq(3,8),(7,8),} \\
& {\left[v_{8}, v_{3}\right]=v_{11}, \quad\left[v_{8}, v_{7}\right]=v_{15} .}
\end{aligned}
$$

Proof. Every $y \in H^{*}\left(F_{4} ; Z_{3}\right)$ can be written as

$$
y=x+a_{1} x_{11}+a_{2} x_{15}+a_{3} x_{11} x_{15}
$$

with $x, a_{i}(1 \leqq i \leqq 3) \in \Lambda_{3}\left(x_{3}, x_{7}\right) \otimes Z_{3}\left[x_{3}\right] /\left(x_{8}^{3}\right)$. If we put

$$
\begin{aligned}
& h^{*}\left(a_{1} x_{11}\right)=h^{*}\left(a_{1}\right)\left(x_{11} \otimes 1+1 \otimes x_{11}\right)+b_{1}, \\
& h^{*}\left(a_{2} x_{15}\right)=h^{*}\left(a_{2}\right)\left(x_{15} \otimes 1+1 \otimes x_{15}\right)+b_{2}, \\
& h^{*}\left(a_{3} x_{11} x_{15}\right)=h^{*}\left(a_{3}\right)\left(x_{11} \otimes 1+1 \otimes x_{11}\right)\left(x_{15} \otimes 1+1 \otimes x_{15}\right)+b_{3},
\end{aligned}
$$

then $h^{*}(y)-\sum b_{j}$ is symmetric in the sense of [5], p.283. In case $y$ is monomial, put $y=x_{i_{1}} \cdots x_{i_{r}} x_{8}^{\varepsilon}(0 \leqq \varepsilon \leqq 2)$, then

$$
h^{*}(y)-\sum b_{j}=\Pi\left(x_{i_{s}} \otimes 1+1 \otimes x_{i_{s}}\right) \cdot\left(x_{8} \otimes 1+1 \otimes x_{8}\right)^{\varepsilon} .
$$

If we write $b_{j}$ explicitly like $b_{1}=h^{*}\left(a_{1}\right) \cdot\left(x_{8} \otimes x_{3}\right)$, etc., then we see easily that

$$
\begin{aligned}
& <b_{j}, v_{i_{1}, \ldots, i_{r-1}} \otimes v_{i_{r}}>=0 \\
& <b_{j}, v_{i_{1}}, \ldots, i_{r} \otimes v_{8}>=0 \\
& <b_{j}, v_{i_{1}, \ldots, i_{r} ; 1} \otimes v_{8}>=0
\end{aligned}
$$

for any subsequence $\left\{i_{1}, \ldots, i_{r}\right\} \subset\{3,7,11,15\}$ and $j=1,2,3$, and that 


$$
\begin{array}{ll}
<b_{1}, v_{i} \otimes v_{j}>=0 & \text { if }(i, j) \neq(8,3), \\
<b_{2}, v_{i} \otimes v_{j}>=0 & \text { if }(i, j) \neq(8,7), \\
<b_{3}, v_{i} \otimes v_{j}>=0 & \text { for all }(i, j) .
\end{array}
$$

Using (5) and (6) we can see that

$$
\begin{aligned}
& v_{i_{1}, \ldots, i_{r}}=v_{i_{1}}, \ldots, i_{r-1} \vee v_{i_{r}} \\
& v_{i_{1}, \ldots, i_{r} ; 1}=v_{i_{1}, \ldots, i_{r}} \vee v_{8} \\
& v_{i_{1}, \ldots, i_{r} ; 2}=2 v_{i_{1}, \ldots, i_{r} ; 1} \vee v_{8},
\end{aligned}
$$

whence by an induction on $r$ we have

$$
\begin{aligned}
& v_{i_{1}, \ldots, i_{r}}=v_{i_{1}} \vee \cdots \vee v_{i_{r}} \\
& v_{i_{1}, \ldots, i_{r} ; 1}=v_{i_{1}} \vee \cdots \vee v_{i_{r}} \vee v_{8} \\
& v_{i_{1}, \ldots, i_{r} ; 2}=2 v_{i_{1}} \vee \cdots \vee v_{i_{r}} \vee v_{8} \vee v_{8}
\end{aligned}
$$

for any $\left\{i_{1}, \ldots, i_{r}\right\} \subset\{3,7,11,15\}$. Hence $v_{i}, i=3,7,11,15,8$, form a 3-simple system of generators.

(7) and the fact that $h^{*}(y)-\sum b_{j}$ is symmetric show that

$$
\left[v_{i}, v_{i}\right]=0 \text { for }(i, j) \neq(8,3),(8,7) \text { and } i \geqq j \text {. }
$$

In particular we have $v_{i} \vee v_{i}=0$ for $i$ odd.

The proofs of the last two relations are entirely the same with the corresponding part of the proof of [5], Théorème 16.4, and are omitted. (q.e.d.)

\section{Chapter II. On some homology relations of loop spaces of compact exceptional groups}

\section{$\S 1$. Preliminaries}

1. Let $K$ be a compact connected and simply connected Lie group. BottSamelson [10] described a homology basis of $H_{*}(\Omega K ; Z)$ ( $\Omega K$ is the loop space of $K$ ) by making use of $K$-cycles.

Let $T \subset K$ be a fixed maximal torus in $K, R$ be the universal covering group of $T$ and $\eta: R \rightarrow T$ be the covering map. Further we denote by $D$ the diagram defined on $R$.

By a singular plane of codimension 1 in $D$ we mean a pair $(\theta, n)$ of a root form $\theta$ and an integer $n$. It is oriented and $(\theta, n)$ and $(-\theta,-n)$ are distinguished. Let $P=\left\{p_{1}, \ldots, p_{k}\right\}$ be a finite ordered set of singular planes in $D$ of codimension 1 . We use the following notations due to [10]: $\bar{p}_{i}=\eta\left(p_{i}\right)$, 
$K\left(p_{i}\right)=$ the connected centralizer of $\cdot \bar{p}_{i}, W(P)=\prod_{1}^{k} K\left(p_{i}\right)$. Then the $K$-cycle $\Gamma(P)$ associated with $P$ is defined by $\Gamma(P)=W(P) / T^{k}$ where the right translations of $T^{k}$ on $W(P)$ are defined by

$$
\left(x_{1}, \ldots, x_{k}\right) \cdot\left(t_{1}, \ldots, t_{k}\right)=\left(x_{1} t_{1}, t_{1}^{-1} x_{1} t_{2}, \ldots, t_{k-1}^{-1} x_{k} t_{k}\right)
$$

for $\left(x_{1}, \ldots, x_{k}\right) \in W(P)$ and $\left(t_{1}, \ldots, t_{k}\right) \in T^{k}$.

Later we need $K$-cycles $\Gamma(P)$ without the restriction of codim $p_{i}=1$ to obtain some relations between homology classes in $H_{*}(\Omega K ; Z)$ expressed by $K$-cycles. They are called here the general $K$-cycles to distinguish from the original $K$-cycles.

The $K$-cycle $\Gamma(P)$ admits an iterated fibration with cross-sections. The base space is $K\left(p_{1}\right) / T$ and the successive fibres are $K\left(p_{2}\right) / T, \ldots, K\left(p_{k}\right) / T . K\left(p_{i}\right) / T$ are orientable, whence $I^{\prime}(P)$ is orientable. If $\operatorname{codim} p_{i}=1$, then $K\left(p_{i}\right) / T$ is a 2 -sphere and has a canonical orientation determined by the root vector of $p_{i}$ as in [10], Chap. II, $\S 3$. Hence the original $K$-cycle has a natural orientation. The orientation of a general $K$-cycle $\Gamma(P)$ is determined depending on the choice of the orientations of $K\left(p_{i}\right) / T$ with $\operatorname{codim} p_{i} \geqq 2$.

2. Let $P^{\prime}=\left\{p_{i_{1}}, \ldots, p_{i_{r}}\right\}$ be a subsequence of $P$. We imbed $\Gamma\left(P^{\prime}\right)$ as a submanifold of $\Gamma(P)$. Let $i: W\left(P^{\prime}\right) \rightarrow W(P)$ be an inclusion defined by

$$
\begin{aligned}
\pi_{t} \circ i\left(x_{1}, \ldots, x_{r}\right) & =x_{t} & & \text { if } t \in\left\{i_{1}, \ldots, i_{r}\right\} \\
& =e & & \text { if } t \notin\left\{i_{1}, \ldots, i_{r}\right\}
\end{aligned}
$$

for $\left(x_{1}, \ldots, x_{r}\right) \in W\left(P^{\prime}\right)$, where $\pi_{t}: W(P) \rightarrow K\left(p_{t}\right)$ is the natural projection onto the $t$-th factor and $e$ is the neutral element of the group. Let $h: T^{r} \rightarrow T^{k}$ be a homomorphism defined by

$$
\begin{aligned}
\pi_{t} \circ h\left(t_{1}, \ldots, t_{r}\right) & =e \quad \text { if } t<i_{1} \\
& =t_{1} \quad \text { if } i_{1} \leqq t<i_{2} \\
& \cdots \ldots \ldots \\
& =t_{s} \quad \text { if } i_{s} \leqq t<i_{s+1} \text { for } s<r \\
& =t_{r} \quad \text { if } i_{r} \leqq t
\end{aligned}
$$

for $\left(t_{1}, \ldots, t_{r}\right) \in T^{r}$ where $\pi_{t}: T^{k} \rightarrow T$ is the natural projection onto the $t$-th factor. Then $i$ is clearly a boundle map relative to the homomorphism $h$ and induces an injection 


$$
\bar{i}: \Gamma\left(P^{\prime}\right) \subset \Gamma(P) .
$$

This imbedding may be considered as a canonical one, and $\Gamma\left(P^{\prime}\right)$, oriented naturally or in a suitable way, gives an integral cycle in $\Gamma(P)$. The submanifold $\Gamma\left(P^{\prime}\right)$ is called a sub $K$-cycle in $\Gamma(P)$.

3. $H_{*}(\Gamma(P) ; Z)$ has no torsion, and in case $\Gamma(P)$ being an original one BottSamelson [10], Chap. II, Prop. 4.2, determined the cohomology ring $H *(\Gamma(P)$; $Z)$. Sub $K$-cycles $\Gamma\left(p_{i}\right)$ in $\Gamma(P)$ associated with a single plane $p_{i}(1 \leqq i \leqq k)$ form a basis of $H_{2}(\Gamma(P) ; Z)$. Let $\left\{x_{i}\right\}$ be the dual basis of $H^{2}(\Gamma(P) ; Z)$. Then by [10]. Chap. II, Prop. 4.2, we see easily that $x_{j_{1}} \cdots x_{j_{s}}, j_{1}<j_{2}<\cdots<j_{s}$, form an additive basis of $H *(\Gamma(P) ; Z)$. On the other hand $\overline{i *}\left(x_{j_{1}} \cdots x_{j_{s}}\right)=$ the top dimensional generator of $H *\left(T\left(P^{\prime}\right) ; Z\right)$ or zero according as $\left\{j_{1}, \ldots\right.$, $\left.j_{s}\right\}=\left\{i_{1}, \ldots, i_{r}\right\}$ or not. From these we see easily that sub $K$-cycles in $\Gamma(P)$ form an additive basis of $H_{*}(\Gamma(P) ; Z)$ when $\Gamma(P)$ is an original one. In case of a general $K$-cycle the description of the homology basis is more involved.

4. For any $K$-cycle $I^{\prime}(P)$ there are associated a homology class $P_{*}$ in $H_{*}(\Omega K ; Z)[9,10]$. For the definition of a chain $c=\left\{c_{0}, c_{1}, \ldots, c_{k}\right\}$ subject to $P$ we refer to $[9], \S 3$. For any chanin $c$ subject to $P$ we have a map

$$
f_{P}^{c}: \Gamma(P) \rightarrow \Omega K .
$$

$P_{*}$ is defined by $P_{*}=f_{P^{*}}^{c}$ (the fundamental class of $\left.I^{\top}(P)\right) . \quad P_{*}$ is determined independently of $c$. These constructions are valid also for general $K$-cycles. The only thing we should take care of is the orientation of $\Gamma(P)$ as we mentioned already.

5. Let $P=\left\{p_{2}, \ldots, p_{k}\right\}$ be an ordered set of $k-1$ singular planes of condimension 1 in $D$. Let $\theta_{1}$ and $\theta_{2}$ be two roots of $K$ such that they have the same length and $\left(\theta_{1}, \theta_{2}\right)<0$. Then $\theta_{3}=\theta_{1}+\theta_{2}$ is a root of $K$. Let $n$ be an integer and we put $q_{1}=\left(\theta_{1}, n\right), q_{2}=\left(\theta_{2}, 0\right), q_{3}=\left(\theta_{3}, n\right)$ and $p_{1}=q_{1} \cap q_{2}$. Then $q_{1}, q_{2}$ and $q_{3}$ contain $p_{\mathrm{i}}$. Put $P^{\prime}=\left\{p_{1}, P\right\} . \quad \Gamma\left(P^{\prime}\right)$ is a general $K$-cycle containing $\Gamma(P)$ as a sub $K$-cycle. Put $P_{i}=\left\{q_{i}, P\right\} \quad(i=1,2,3)$. The inclusions $K\left(q_{i}\right) \subset K\left(p_{1}\right)$ induce inclusions $W\left(P_{i}\right) \subset W\left(P^{\prime}\right)$, which are bundle maps and induce in turn imbeddings

$$
\Gamma\left(P_{i}\right) \subset \Gamma\left(P^{\prime}\right) \quad \text { for each } i=1,2,3 .
$$

The semi-simple part of $K\left(p_{1}\right)$ is of type $A_{2}$ and is denoted by $A_{2}$, which 
have $\pm \theta_{i}(i=1,2,3)$ as roots. $A_{2} / T^{\prime} \approx K\left(p_{1}\right) / T$ canonically where $T^{\prime}=A_{2} \cap T$ is a maximal torus in $A_{2}$. Let $\tau_{i}(i=1,2,3)$ be root vectors associated with $\theta_{i}$ in the sense of Stiefel [17], i.e., $\tau_{i}$ is perpendicular to $\theta_{i}$ and $\theta_{i}\left(\tau_{i}\right)=2$. $\tau_{i}$ defines an integral cycle in $H_{1}\left(T^{\prime}\right)$ with a relation $\tau_{3}=\tau_{1}+\tau_{2}$. 2-spheres $K\left(q_{i}\right) / T$ in $A_{2} / T^{\prime}$ represent 2-cycles $y_{i}$ of $H_{2}\left(A_{2} / T^{\prime}\right)$ such that their homology transgression images in the fibration $\left(A_{2}, A_{2} / T^{\prime}, T^{\prime}\right)$ are $\tau_{i}$. Since this transgression defined on $H_{2}\left(A_{2} / T^{\prime}\right)$ is injective, we have the relation

$$
y_{3}=y_{1}+y_{1} .
$$

$\Gamma\left(P^{\prime}\right)$ is fibred with $\Gamma(P)$ as its fibre and with $A_{3} / T^{\prime}$ as its base space. The associated homology spectral sequence over $Z$ is collapsed because the fibre and the base space have trivial homology groups in odd degrees. The integral cycle $\Gamma\left(P_{i}\right)$ represents $y_{i} \otimes P_{*}$ in the $E_{\infty}$ term for each $i=1,2,3$, where $P_{*}$ denotes the fundamental class of the homology group of the fibre. (1) implies that

$$
y_{3} \otimes P_{*}=y_{1} \otimes P_{*}+y_{2} \otimes P_{*} \quad \text { in } E_{\infty} .
$$

Since $P_{*}$ is a homology class of the fibre with the highest degree, (1') implies in turn that

$$
\Gamma\left(P_{3}\right)_{*}=\Gamma\left(P_{1}\right)_{*}+\Gamma\left(P_{2}\right)_{*}
$$

where $\Gamma\left(P_{i}\right)_{*}$ denote the classes in $H_{*}\left(\Gamma\left(P^{\prime}\right) ; Z\right)$ represented by the integral cycles $\Gamma\left(P_{i}\right)$ in $\Gamma\left(P^{\prime}\right)$.

Proposition 1. $P_{1}, P_{2}$ and $P_{3}$ are defined as above, then

$$
P_{3 *}=P_{1 *}+P_{2 *} \quad \text { in } H_{2 k}(\Omega K ; Z) \text {. }
$$

Proof. Let $c$ be a chain subject to $P^{\prime}$. Then $c$ is also subject to $P_{i}, i=1$, 2, 3. And

$$
f_{P_{i}}^{c}=f_{P^{\prime}}^{c} \mid P_{i} \quad \text { for each } i=1,2,3 .
$$

Then (2) and the definition of $P_{i *}$ imply the conclusion of this proposition.

(q.e.d.)

6. Let $W$ be the Weyl group of $K$ operated in $R$ or in $T$. An element $w \in W$ transforms an ordered set $P=\left\{p_{1}, \ldots, p_{k}\right\}$ of singular planes to $w P$ $=\left\{w p_{1}, \ldots, w p_{k}\right\}$.

Let a be a representative of $w$ in the normalizer $N(T)$ of $T$. The inner 
automorphism $\varphi_{a}: K \rightarrow K$ defined by $a$, maps $K\left(p_{i}\right)$ onto $K\left(w p_{i}\right)$ as is easily seen, and induces $\bar{\varphi}_{a}: W(P) \rightarrow W(w P)$ defined by $\bar{\varphi}_{a}=\varphi_{a} \times \cdots \times \varphi_{a}$. This is a bundle map relative to a homomorphism $\bar{w}: T^{k} \rightarrow T^{k}$ defined by the diagonal action of $w$, whence we have an induced map

$$
\varphi_{a \sharp}: \Gamma(P) \rightarrow \Gamma(w P) .
$$

$\varphi_{a *}: H_{*}\left(I^{\prime}(P)\right) \rightarrow H_{*}\left(I^{\prime}(w P)\right)$ depends only on $w$ and does not depend on the choice of $a$. The inner automorphism $\varphi_{a}$ induces a map $\phi_{a}: \Omega K \rightarrow \Omega K$. Since $K$ is connected, $\psi_{a}$ is homotopic to the identity map and $\psi_{a *}: H(\Omega K) \rightarrow H(\Omega K)$ is equal to the identity.

Let $c$ be a chain subject to $P$, then $w c$ is a chain subject to $w P$. Now the following diagram

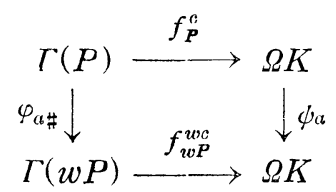

is clearly commutative, whence we have the

Proposition 2. $P_{*}=(w P)_{*} \quad$ for any $w \in W$.

In case $\Gamma(P)$ is a general $K$-cycle we must take care of the orientations of $I^{\cdot}(P)$ and $\Gamma(w P)$ in the above proposition.

7. Let $P=\left\{p_{1}, \ldots, p_{k}\right\}$ be an ordered set of singular planes with codim $p_{j}=1$ except a plane $p_{i}$. Assume that $p_{i}$ has codimension 2 and $p_{i}=\left(\theta_{1}, n\right)$ $\cap\left(\theta_{2}, o\right)$ such that $\theta_{1}$ and $\theta_{2}$ have the same length and $\left(\theta_{1}, \theta_{2}\right)<0$. Then the semisimple part of $K\left(p_{i}\right)$ is of type $A_{2}$ and is denoted by $A_{2}$ as in No. 1.5. Put $\theta_{3}=\theta_{1}+\theta_{2}$, then the plane $\left(\theta_{3}, n\right)$ contains $p_{i}$. The Weyl group $W\left(A_{2}\right)$ of $A_{2}$ is a subgroup of the Weyl group $W(K)$ of $K$. Let $R_{2} \in W\left(A_{2}\right)$ be the reflection across $\left(\theta_{2}, 0\right)$. $R_{2}$ maps $\left(\theta_{1}, n\right)$ onto $\left(\theta_{3}, n\right)$ and vice versa. Hence $R_{2}$ keeps $p_{i}$ invariant.

If we fix an orientation of $K\left(p_{i}\right) / T$, then the orientation of $\Gamma^{\prime}(P)$ as well as the orientation of $\Gamma\left(R_{2} P\right)$ is determined. Now $R_{2}$ reverses the orientation of $T$, whereas $\varphi_{a}$ preserves the orientation of $K\left(p_{i}\right)$ where $\varphi_{a}$ is an inner automorphism of $K\left(p_{i}\right)$ defined by a representative $a$ of $R_{2}$ in $N\left(T \cap A_{2}\right)$. Hence the induced map $\varphi_{a \sharp}: K\left(p_{i}\right) / T \rightarrow K\left(p_{i}\right) / T$ reverses the orientation. Hence by the Prop. 2 and the remark at the end of No. 2.6 we see that $P_{*}=-\left(R_{2} P\right)_{*}$. 
In particular, if $R_{2}$ keeps planes $p_{j}$ invariant of $j \neq i$, i.e., $P=R_{2} P$, then we see that $P_{*}=0$.

Proposition 3. Let $P$ be as above and assume that $\theta_{2}$ is orthogonal to all roots of $p_{j}$ for $j \neq i$. Then $P_{*}=0$.

8. Here we give a modified description of an original $K$-cycle $\Gamma(P)$ which is convenient in the next No.

Let $P=\left\{p_{1}, \ldots, p_{k}\right\}$ be an ordered set of $k$ singular planes of codimension 1. The semisimple part of $K\left(p_{i}\right)$ is a 3 -sphere denoted by $S^{3}\left(p_{i}\right)$ whose maximal torus $S^{1}\left(p_{i}\right)=S^{3}\left(p_{i}\right) \cap T$ is a circle perpendicular to $\bar{p}_{i}$. We remark that for any $t \in T$ the inner automorphism defined by $t$ maps $S^{3}\left(p_{i}\right)$ into itself.

Put $W^{\prime}(P)=\prod_{1}^{k} S^{3}\left(p_{i}\right)$ and $T^{\prime}(P)=\prod_{1}^{k} S^{1}\left(p_{i}\right)$. Let $T^{\prime}(P)$ operate on $W^{\prime}(P)$ by

$$
\begin{gathered}
\left(x_{1}, \ldots, x_{k}\right) \cdot\left(t_{1}, \ldots, t_{k}\right)=\left(x_{1} t_{1}, t_{1}^{-1} x_{2} t_{1} t_{2}, \ldots,\right. \\
\left.t_{1}^{-1} \cdots t_{i-1}^{-1} x_{i} t_{1} \cdots t_{i}, \ldots, t_{1}^{-1} \cdots t_{k-1}^{-1} x_{k} t_{1} \cdots t_{k}\right)
\end{gathered}
$$

for $\left(x_{1}, \ldots, x_{k}\right) \in W^{\prime}(P)$ and $\left(t_{1}, \ldots, t_{k}\right) \in T^{\prime}(P)$. Then $W^{\prime}(P)$ is a principal $T^{\prime}(P)$ bundle. Let $f: T^{\prime}(P) \rightarrow T^{k}$ be a homomorphism defined by

$$
f\left(t_{1}, \ldots, t_{k}\right)=\left(t_{1}, t_{1} t_{2}, \ldots, t_{1} \cdots t_{i}, \ldots, t_{1} \cdots t_{k}\right)
$$

for $\left(t_{1}, \ldots, t_{k}\right) \in T^{\prime}(P)$. Then the natural inclusion $W^{\prime}(P) \rightarrow W(P)$ is clearly a bundle map relative to $f$ and induces a homeomorphism of base spaces, i.e., $W^{\prime}(P) / T^{\prime}(P) \approx I(P)$.

9. Here we assume that the group $K$ is simple and simply laced, i.e., all roots of $K$ have the same length. In an original $K$-cycle $\Gamma(P)$ with $P=\left\{p_{1}\right.$, $\left.\ldots, p_{k}\right\}$ let us assume that two successive planes $p_{i}$ and $p_{i+1}$ are orthogonal to each other and put $P^{\prime}=\left\{p_{1}, \ldots, p_{i-1}, p_{i+1}, p_{i}, p_{i+2}, \ldots, p_{k}\right\}$.

Proposition 4.

$$
P_{*}=P_{*}^{\prime} \text {. }
$$

Proof. The roots of $p_{i}$ and $p_{i+1}$ are denoted by $\theta_{i}$ and $\theta_{i+1}$. Since $K$ is simply laced, $\theta_{i} \pm \theta_{i+1}$ are not roots. This implies that the semisimple part of $K(q), q=p_{i} \cap p_{i+1}$, is of type $A_{1} \times A_{1}$, and the direct factors are $S^{3}\left(p_{i}\right)$ and $S^{3}\left(p_{i+1}\right)$ respectively. Hence $S^{3}\left(p_{i}\right)$ and $S^{3}\left(p_{i+1}\right)$ are elementwise commutative in $K$.

Let $\chi: W^{\prime}(P) \rightarrow W^{\prime}\left(P^{\prime}\right)$ be a map defined by $\chi\left(x_{1}, \ldots, x_{k}\right)=\left(x_{1}, \ldots\right.$, $\left.x_{i-1}, x_{i+1}, x_{i}, x_{i+2}, \ldots, x_{k}\right)$ for $\left(x_{1}, \ldots, x_{k}\right) \in W^{\prime}(P) . \quad \bar{\chi}=\chi \mid T^{\prime}(P): T^{\prime}(P)$ 
$\rightarrow T^{\prime}\left(P^{\prime}\right)$ is a homomorphism and $\%$ is a bundle map relative to $\bar{\chi}$ inducing a homeomorphism of base spaces

$$
\tilde{\chi}: \Gamma(P) \approx \Gamma\left(P^{\prime}\right) .
$$

$\tilde{\chi}$ is orientation preserving since $\tilde{\chi}^{*}$ transforms the cohomology fundamental class $x_{1} \cdots x_{k}$ to the cohomology fundamental class as is easily seen.

Let $c=\left\{c_{0}, \ldots, c_{k-1}\right\}$ be a chain subject to $P^{\prime \prime}=\left\{p_{1}, \ldots, p_{i-1}, q, p_{i+2}\right.$, $\left.\ldots, p_{k}\right\}$. $c$ may be considered as a chain subject to $P$ or to $P^{\prime}$ with $(i+1)$-th polygon collapsed to a point. Since $S^{3}\left(p_{i}\right)$ and $S^{3}\left(p_{i+1}\right)$ are elementwise commutative in $K$ we see easily that the diagram

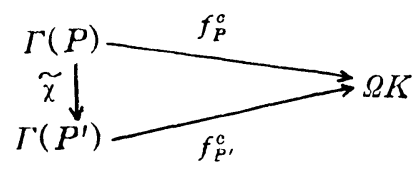

is commutative. Hence $P_{*}=P_{*}^{\prime}$.

10. Let $I^{\prime}(P)$ be an original $K$-cycle with $P=\left\{p_{1}, \ldots, p_{k}\right\}$. Further, assume that $p_{i}=p_{i+1}$. Then

Proposition 5. $\quad P_{*}=0$.

Proof. Put $P^{\prime}=\left\{p_{1} \cdots \stackrel{i}{\vee} \cdots p_{k}\right\}$ by deleting $p_{i}$ from $P$. Let

$$
\alpha: K\left(p_{i}\right) \times K\left(p_{i+1}\right) \rightarrow K\left(p_{i+1}\right)
$$

be the map defined by the multiplication in the group.

$$
\bar{\alpha}=\imath \times \cdots \times \imath \times \alpha \times \imath \times \cdots \times \imath: W(P) \rightarrow W\left(P^{\prime}\right)
$$

is a bundle map relative to $\bar{\alpha} \mid T^{k}$, where ( is identity map, and induces a may

$$
\tilde{\alpha}: \Gamma(P) \rightarrow \Gamma\left(P^{\prime}\right) .
$$

Let $c$ be a chain subject to $P^{\prime} . \quad c$ is also subject to $P$ with $(i+1)$-th polygon collapsed to a point. The diagram

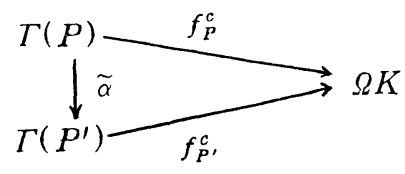

is clearly commutative and 
$\widetilde{a}_{*}$ (the fundamental class of $\left.\Gamma(P)\right)=0$

since $\operatorname{dim} \Gamma(P)>\operatorname{dim} \Gamma\left(P^{\prime}\right)$. Hence $P_{*}=0$.

(q.e.d.)

11. Let $\widetilde{f}$ be a fundamental chamber in $D$, i.e.,

$$
\widetilde{F}=\left\{X \subseteq R ; \varphi_{i}(X)>0 \quad \text { for all } 1 \leqq i \leqq l\right\}
$$

where $\varphi_{i}, 1 \leqq i \leqq l$, are simple roots of a fundamental system of roots of $K$.

$\tilde{f}$ is subdivided in cells by singular planes in it. Let $\phi$ be the 1 -skeleton of the dual subdivision of $\mathfrak{F}$, called graph. To each vertex of $\phi$ there corresponds a cell of $\mathfrak{F}$ and to each edge of $\mathfrak{F}$ there corresponds a cell in a singular plane $(\theta, n)$ in $\widetilde{\mho}$. Let $\mu$ denote the dominant root of the fundamental system of roots $\left\{\varphi_{i}\right\} . \quad(\mu, 1)$ is the nearest to the origin among the singular planes in $\mathfrak{F}$ so that $\{X \in \mathfrak{F} ; \mu(X)<1\}$ is a cell in $\mathfrak{F}$, i.e., the fundamental cell in $\mathfrak{F}$ denoted by $\Delta_{\mathfrak{F}}$. $v_{\mathfrak{F}}$ is the vertex dual to $\Delta_{\mathfrak{F}}$. The dual vertex of a cell $\Delta$ is denoted by $v_{\perp}$.

Bott-Samelson [10], Chap. II, Prop. 9.1, described a homology basis of $H_{*}(\Omega K ; Z)$ using $K$-cycles. To each vertex $v \in \phi$ there corresponds an element $P_{*}$ of this basis such that $P$ consists of the planes in a suitable path connecting $v_{\Delta}$ to $v_{\widetilde{\zeta}}$ in that order. Actually it would yield some difficulties to determine the path carrying the generator. Nevertheless, in cases discussed in the next section this point is solved by the Prop. 4.

By $\phi_{i}$ we denote the subgraph of $\phi$ containing all vertices and edges which are connected to $v_{\widetilde{⿰}}$ through, at most, $i$ successive edges. $\phi_{i}$ is sufficient to determine the homology basis of $\Omega K$ in deg. $\leqq 2 i$.

$\S 2$. Homology basis in low degrees of loop spaces of $F_{4}, E_{6}, E_{7}, E_{8}$

1. The Schläfli figure of $F_{4}$ is as follows :

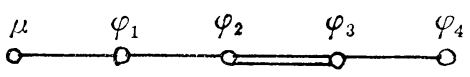

where $\varphi_{i}, 1 \leqq i \leqq 4$, is a fundamental system of roots of $F_{4}$ and $\mu$ is the dominant root of the fundamental system. $\phi_{5}\left(F_{4}\right)$ is described as follows:

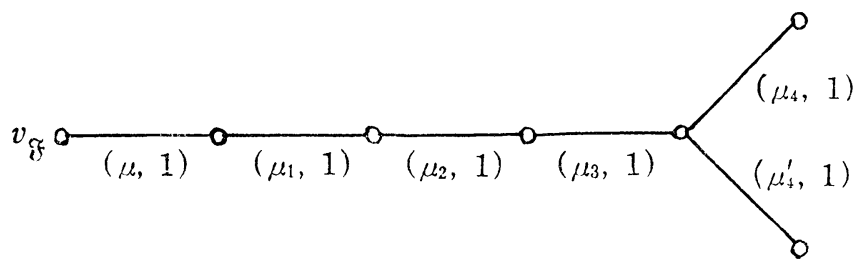


where $\mu_{i}=\mu_{i-1}-\varphi_{i}$ for $1 \leqq i \leqq 4 \quad\left(\mu_{0}=\mu\right)$ and $\mu_{4}^{\prime}=\mu_{3}-\varphi_{3}$. This is obtained without difficulties by a succession of reflections of cells on some incident singular planes starting from $\Delta \mathfrak{r}$.

Then the homology basis of $H_{*}\left(\Omega F_{4} ; Z\right)$ for deg. $\leqq 10$ is given by

where

$$
P_{1 *}, P_{2 *}, P_{3 *}, P_{4 *}, P_{5 *}^{1} \text { and } P_{5 *}^{1} \text {, }
$$

$$
\begin{aligned}
& P_{i}=\left\{\left(\mu_{i-1}, 1\right),\left(\mu_{i-2}, 1\right), \ldots,(\mu, 1)\right\} \quad \text { for } 1 \leqq i \leqq 4, \\
& P_{5}^{1}=\left\{\left(\mu_{1}, 1\right),\left(\mu_{3}, 1\right), \ldots,(\mu, 1)\right\}, \\
& P_{5}^{2}=\left\{\left(\mu_{4}^{\prime}, 1\right),\left(\mu_{3}, 1\right), \ldots,(\mu, 1)\right\}
\end{aligned}
$$

and the double of the lower suffixes denote the dimensions of the corresponding $F_{4}$-cycles.

2. The Schläfli figure of $E_{6}$ is as follows :

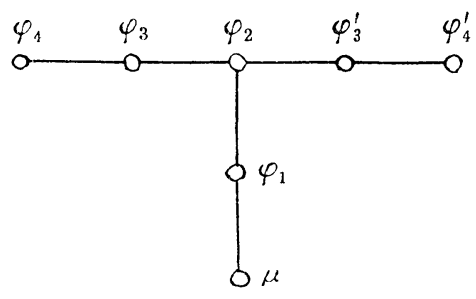

where $\varphi_{i}$ and $\varphi_{i}^{\prime}$ are simple roots of a fundamental system of roots of $E_{6}$ and $\mu$ is the dominant root. $\phi_{5}\left(E_{6}\right)$ is described as follows:

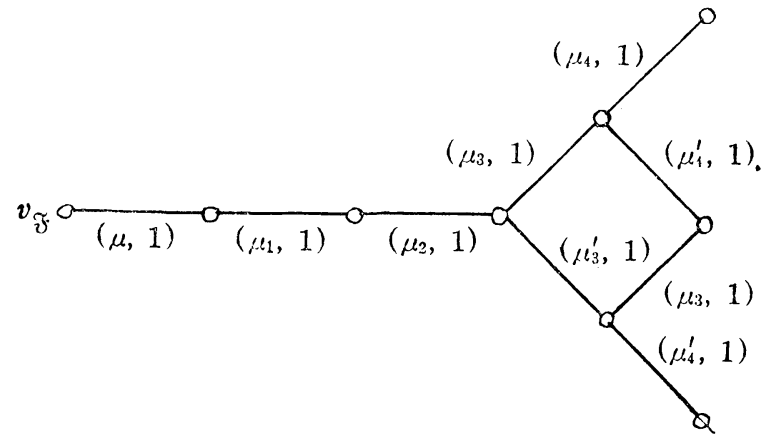

where $\mu_{i}=\mu_{i-1}-\varphi_{i}$ for $1 \leqq i \leqq 4\left(\mu_{0}=\mu\right)$ and $\mu_{3}^{\prime}=\mu_{2}-\varphi_{3}^{\prime}, \mu_{1}^{\prime}=\mu_{3}^{\prime}-\varphi_{4}^{\prime}$.

Notations of ordered sets of singular planes:

$$
\begin{aligned}
& P_{i}=\left\{\left(\mu_{i-1}, 1\right),\left(\mu_{i-2}, 1\right), \ldots,(\mu, 1)\right\} \quad \text { for } 1 \leqq i \leqq 3, \\
& P_{4}^{1}=\left\{\left(\mu_{3}, 1\right), P_{3}\right\}, P_{4}^{2}=\left\{\left(\mu_{3}^{\prime}, 1\right), P_{3}\right\}, \\
& P_{5}^{1}=\left\{\left(\mu_{4}, 1\right),\left(\mu_{3}, 1\right), P_{3}\right\}, \quad P_{5}^{2}=\left\{\left(\mu_{4}^{\prime}, 1\right),\left(\mu_{3}^{\prime}, 1\right), P_{3}\right\}, \\
& P_{5}^{3}=\left\{\left(\mu_{3}^{\prime}, 1\right),\left(\mu_{3}, 1\right), P_{3}\right\}, \quad P_{5}^{4}=\left\{\left(\mu_{3}, 1\right),\left(\mu_{3}^{\prime}, 1\right), P_{3}\right\} .
\end{aligned}
$$


Here we remark that $\mu_{3}$ and $\mu_{3}^{\prime}$ are orthogonal to each other, then by the Prop. 4 we see that $P_{5 *}^{3}=P_{5 *}^{4}$. Hence the homology basis of $H_{*}\left(\Omega E_{6} ; Z\right)$ in deg. $\leqq 10$ is given by

$$
P_{1 *}, P_{2 *}, P_{3 *}, P_{4 *}^{1}, P_{4 *}^{2}, P_{5 *}^{1}, P_{5 *}^{2} \text { and } P_{5 *}^{3}
$$

where the double of lower suffixes denote degrees of these generators.

3. The Schläfli figure of $E_{\bar{i}}$ is as follows :

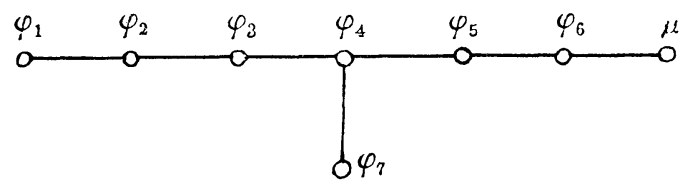

where $\mu$ is the dominant root. $\phi_{7}\left(E_{7}\right)$ is described as follows :

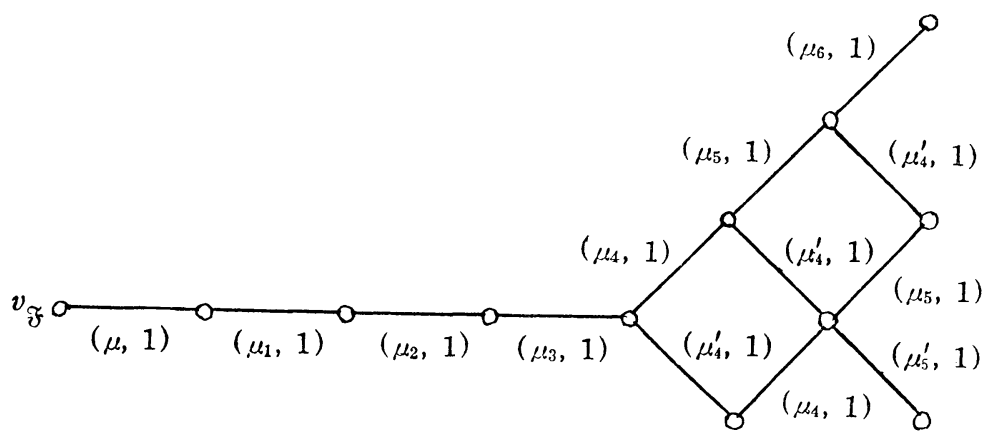

where $\mu_{i}=\mu_{i-1}-\varphi_{7-i}$ for $1 \leqq i \leqq 6\left(\mu_{0}=\mu\right)$ and $\mu_{4}^{\prime}=\mu_{3}-\varphi_{7}, \mu_{5}^{\prime}=\mu_{1}-\varphi_{7}$.

Notations of ordered sets of singular planes :

$$
\begin{array}{lll}
P_{i}=\left\{\left(\mu_{i-1}, 1\right),\left(\mu_{i-2}, 1\right), \ldots,(\mu, 1)\right\} & \text { for } 1 \leqq i \leqq 4, \\
P_{5}^{1}=\left\{\left(\mu_{4}, 1\right), P_{4}\right\}, & P_{5}^{2}=\left\{\left(\mu_{1}^{\prime}, 1\right), P_{4}\right\}, & \\
P_{6}^{1}=\left\{\left(\mu_{5}, 1\right), P_{5}^{1}\right\}, & P_{6}^{2}=\left\{\left(\mu_{1}^{\prime}, 1\right), P_{5}^{1}\right\}, & P_{6}^{2}=\left\{\left(\mu_{4}, 1\right), P_{5}^{2}\right\}, \\
P_{7}^{1}=\left\{\left(\mu_{6}, 1\right), P_{6}^{1}\right\}, & P_{7}^{2}=\left\{\left(\mu_{4}^{\prime}, 1\right), P_{6}^{1}\right\}, & P_{7}^{3}=\left\{\left(\mu_{5}^{\prime}, 1\right), P_{6}^{2}\right\}, \\
P_{7}^{4}=\left\{\left(\mu_{5}, 1\right), P_{6}^{2}\right\}, & P_{7}^{5}=\left\{\left(\mu_{5}, 1\right), P_{6}^{3}\right\}, & P_{7}^{6}=\left\{\left(\mu_{5}^{\prime}, 1\right), P_{6}^{3}\right\} .
\end{array}
$$

We remark that $\mu_{4}^{\prime}$ is orthogonal to $\mu_{4}$ and $\mu_{5}$ respectively. Then by the Prop. 4 we see that $P_{6 *}^{2}=P_{6 *}^{3}, P_{7 *}^{2}=P_{7 *}^{4}=P_{7 *}^{5}$ and $P_{7 *}^{3}=P_{7 *}^{6}$. Hence the homology basis of $H_{*}\left(\Omega E_{7} ; Z\right)$ in deg. $\leqq 14$ is given by

$$
P_{i *}(1 \leqq i \leqq 4), P_{5 *}^{1}, P_{5 *}^{2}, P_{6 *}^{1}, P_{6 *}^{2}, P_{7 *}^{1}, P_{7 *}^{2} \text { and } P_{7 *}^{3} .
$$

4. The Schläfli figure of $E_{8}$ is as follows : 


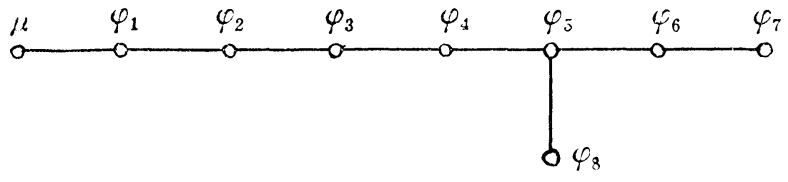

where $\mu$ is the dominant root. $\phi_{\bar{\tau}}\left(E_{8}\right)$ is described as follows:

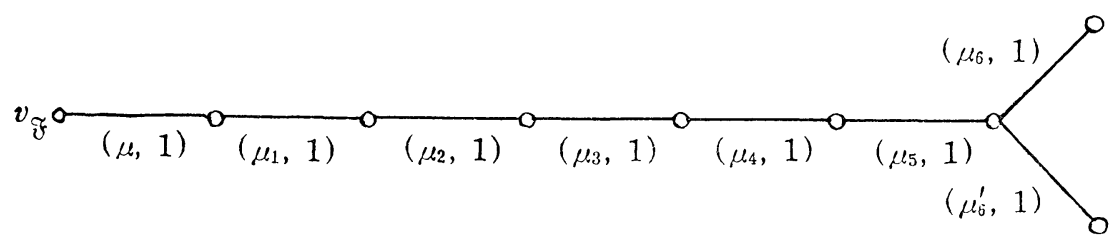

where $\mu_{i}=\mu_{i-1}-\varphi_{i}$ for $1 \leqq i \leqq 6\left(\mu_{0}=\mu_{0}\right)$ and $\mu_{6}^{\prime}=\mu_{5}-\varphi_{8}$.

Notations of ordered sets of singular planes:

$$
\begin{aligned}
& P_{i}=\left\{\left(\mu_{i-1}, 1\right),\left(\mu_{i-2}, 1\right), \ldots,(\mu, 1)\right\} \quad \text { for } 1 \leqq i \leqq 6, \\
& P_{7}^{1}=\left\{\left(\mu_{6}, 1\right), P_{6}\right\}, \quad P_{7}^{2}=\left\{\left(\mu_{6}^{\prime}, 1\right), P_{6}\right\} .
\end{aligned}
$$

The homology basis of $H_{*}\left(\Omega E_{8} ; Z\right)$ in deg. $\leqq 14$ is given by

$$
P_{i *}(1 \leqq i \leqq 6), P_{7 *}^{1} \text { and } P_{7 *}^{2} \text {. }
$$

§ 3. The homology map $\boldsymbol{H}_{*}\left(\Omega F_{4}\right) \rightarrow H_{*}\left(\Omega E_{6}\right)$

1. The inclusion $f: F_{4} \subset E_{6}$ is described by the following table, [16]. Let $T$ be a fixed maximal torus of $E_{6}$ and $T^{\prime}=F_{4} \cap T$. $T^{\prime}$ is a maximal torus of $F_{4}$. Let $R$ and $R^{\prime}$ be the universal covering groups of $E_{6}$ and $F_{4} . \quad R^{\prime}$ is identified with $\eta^{-1} T^{\prime} \subset R$ where $\eta: R \rightarrow T$ is the covering map. The map defined by this identification is denoted by $\bar{f}$. Under the inclusion $f$ every root form of $E_{6}$ determines a root form of $F_{4}$ if restricted to $R^{\prime}$. This correspondence of root forms is given in the above

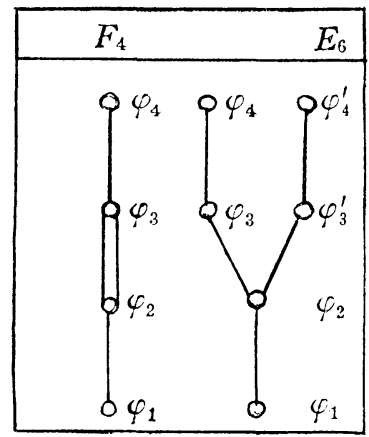
table, i.e.,

$$
\begin{aligned}
& \varphi_{4}\left(E_{6}\right)\left|R^{\prime}=\varphi_{4}^{\prime}\left(E_{6}\right)\right| R^{\prime}=\varphi_{4}\left(F_{4}\right), \\
& \varphi_{3}\left(E_{6}\right)\left|R^{\prime}=\varphi_{3}^{\prime}\left(E_{6}\right)\right| R^{\prime}=\varphi_{3}\left(F_{4}\right), \\
& \varphi_{2}\left(E_{6}\right)\left|R^{\prime}=\varphi_{2}\left(F_{4}\right), \varphi_{1}\left(E_{6}\right)\right| R^{\prime}=\varphi_{1}\left(F_{4}\right) .
\end{aligned}
$$

For every long root of $F_{4}$ there corresponds one root of $E_{6}$ and for every short 
root of $F_{4}$ there correspond two mutually orthogonal roots of $E_{6}$ [16]. These roots of $E_{6}$ are called the associated roots with the given roots of $F_{4}$. The dominant root of $E_{6}$ is associated with the dominant root of $F_{4}$, since

and

$$
\mu\left(E_{6}\right)=2 \varphi_{1}+3 \varphi_{2}+2\left(\varphi_{3}+\varphi_{3}^{\prime}\right)+\left(\varphi_{4}+\varphi_{4}^{\prime}\right)
$$

$$
\begin{aligned}
\mu\left(E_{6}\right) \mid R^{\prime} & =2 \varphi_{1}\left(F_{4}\right)+3 \varphi_{2}\left(F_{4}\right)+4 \varphi_{3}\left(F_{4}\right)+2 \varphi_{4}\left(F_{4}\right) \\
& =\mu\left(F_{4}\right) .
\end{aligned}
$$

By $\tau_{\rho}$ (or $\tau_{\theta}$ ) we denote the root vector corresponding to the root form $\rho$ (or $\theta$ ) of $F_{4}$ (or of $E_{6}$ ) in the sense of Stiefel [17]. If $\rho$ is a long root of $F_{4}$ and $\theta$ is the root of $E_{6}$ associated with $\rho$, then

$$
\bar{f}\left(\tau_{\mathrm{p}}\right)=\tau_{0}
$$

since $\tau_{p}$ is orthogonal to the plane $(\theta, 0)$ in $R$. In particular

$$
\bar{f}\left(\tau_{\nu}\left(F_{4}\right)\right)=\tau_{\mu}\left(E_{6}\right)
$$

where $\mu$ denotes the dominant root of $F_{4}$ or of $E_{6}$. If $\rho$ is a short root of $F_{4}$ and $\theta_{1}, \theta_{2}$ are roots of $E_{6}$ associated with $\rho$, then

$$
\bar{f}\left(\tau_{p}\right)=\tau_{\theta_{1}}+\tau_{0_{2}} \text {. }
$$

This is proved as follows: since $\tau_{p}$ is orthogonal to $\left(\theta_{1}, 0\right) \cap\left(\theta_{2}, 0\right)$ in $R, \vec{f}\left(\tau_{p}\right)$ is a linear combination of $\tau_{\theta_{1}}$ and $\tau_{\theta_{2}}$, say $a \tau_{\theta_{1}}+b \tau_{\theta_{2}}$. Then, since $\theta_{1}$ and $\theta_{2}$ are orthogonal we have $\theta_{1}\left(\bar{f}\left(\tau_{p}\right)\right)=2 a$ and $\theta_{2}\left(\bar{f}\left(\tau_{p}\right)\right)=2 b$. On the other hand $\theta_{i}\left(\bar{f}\left(\tau_{\rho}\right)\right)=\rho\left(\tau_{\rho}\right)=2$. Hence $a=b=1$.

2. The inclusion $f: F_{4} \rightarrow E_{6}$ induces the map of Lie algebras

$$
d f: L\left(F_{4}\right) \rightarrow L\left(E_{6}\right) .
$$

Let

$$
\begin{array}{ll}
L\left(F_{4}\right)=R^{\prime}+\sum \mathfrak{e}_{\alpha} & \left(\operatorname{dim} \mathfrak{e}_{\alpha}=2\right), \\
L\left(E_{6}\right)=R+\sum \mathfrak{e}_{\beta} & \left(\operatorname{dim} \mathfrak{e}_{\beta}=2\right)
\end{array}
$$

be respectively canonical direct sum decompositions of $L\left(F_{4}\right)$ and of $L\left(E_{6}\right)$ into the invariant subspaces under $\operatorname{Ad} T^{\prime}$ and $\operatorname{Ad} T$, where $\alpha$ (or $\beta$ ) runs the positive roots subject to the simple system of $\operatorname{roots}\left\{\varphi_{i}\right\}$ (or $\left\{\varphi_{i}, \varphi_{i}^{\prime}\right\}$ ).

Let $p$ be a singular plane of codimension 1 in $R^{\prime}$ and $\rho$ be the root of $p$. For the sake of simplicity we assume that $\rho$ is positive. By a standard argument (3) and (4) imply that i) if $\rho$ is a long root of $F_{4}$ and $\theta$ is the associated 
root of $E_{6}$, then

$$
d f\left(\mathfrak{e}_{\mathrm{p}}\right) \subset \mathfrak{e}_{\theta}
$$

ii) if $\rho$ is a short root of $F_{4}$ and $\theta_{1}, \theta_{2}$ are the associated roots of $E_{6}$, then

$$
d f\left(\mathfrak{e}_{p}\right) \subset \mathfrak{e}_{\theta_{1}}+\mathfrak{e}_{\theta_{2}} .
$$

Then we see that

$$
f(K(p)) \subset K(q) \quad \text { for each singular plane } p=(\rho, n)
$$

where $q$ is a singular plane of codimension 1 or 2 according as $\rho$ is long or short such that in case $\rho$ is long $q=(\theta, n)$ with $\theta \mid R^{\prime}=\rho$ and in case $\rho$ is short $q=\left(\theta_{1}, n\right) \cap\left(\theta_{2}, n\right)$ with $\theta_{i} \mid R^{\prime}=\rho$. The singular plane $q$ is called the singular plane in $R$ associated with the plane $p$ in $R^{\prime}$.

3. When $p=(\rho, n)$ is a singular plane in $R^{\prime}$ such that $\rho$ is a long root of $F_{4}$ and $q=(\theta, n)$ is the associated plane in $R$, then

$$
f_{p}^{\prime}=f \mid K(p): K(p) \rightarrow K(q)
$$

induces a homeomorphism

$$
f_{p}: K(p) / T^{\prime} \approx K(q) / T
$$

which is orientation preserving by the reason of (3).

When $p=(\rho, n)$ is a singular plane in $R^{\prime}$ such that $\rho$ is a short root of $F_{4}$ and $q=\left(\theta_{1}, n\right) \cap\left(\theta_{2}, n\right)$ is the associated plane, then the semisimple part of $K(q)$ is of type $A_{1} \times A_{1}$. Let $S^{3}\left(\theta_{1}\right)$ and $S^{3}\left(\theta_{2}\right)$ be the semisimple part of $K\left(\theta_{1}\right)$ and $K\left(\theta_{2}\right)$ respectively. Then the semisimple part of $K(q)$ is $S^{3}\left(\theta_{1}\right) \cdot S^{3}\left(\theta_{2}\right)$ and is isomorphic to $S^{3}\left(\theta_{1}\right) \times S^{3}\left(\theta_{2}\right)$ or to $S^{3}\left(\theta_{1}\right) \times{ }_{z_{2}} S^{3}\left(\theta_{2}\right)$ where $Z_{2}$ is identified with the centers of $S^{3}\left(\theta_{i}\right), i=1,2$. Since $f$ is an injection $f \mid S^{3}(\rho)$ is injective, where $S^{3}(\rho)$ is the semisimple part of $K(p)$, which shows that the semisimple part of $K(q)$ is isomorphic to $S^{3}\left(\theta_{1}\right) \times S^{3}\left(\theta_{2}\right)$. Then the map

$$
f \mid S^{3}(\rho): S^{3}(\rho) \rightarrow S^{3}\left(\theta_{1}\right) \times S^{3}\left(\theta_{2}\right)
$$

is the diagonal injection by the reason of (4). And the induced map

$$
f_{p}: K(p) / T^{\prime} \approx S^{2}(\rho) \rightarrow S^{2}\left(\theta_{1}\right) \times S^{2}\left(\theta_{2}\right) \approx K(q) / T
$$

is the diagonal injection of 2 -sphere, where $S^{2}(\rho)=S^{3}(\rho) / S^{1}(\rho)$ and $S^{2}\left(\theta_{i}\right)$ $=S^{3}\left(\theta_{i}\right) / S^{1}\left(\theta_{i}\right)$. 
Now $S^{2}\left(\theta_{i}\right)$ is oriented by $\tau_{\theta_{i}}$ and defines an integral 2-class in $K(q) / T$ for each $i=1,2$. They are homology basis of $H_{*}(K(q) / T ; Z)$. Let $y_{i}, i=1,2$, be the dual basis of $H^{2}(K(q) / T)$, then

$$
f_{q}^{*}\left(y_{i}\right)=x
$$

where $x$ is the cohomology fundamental class of $K(p) / T^{\prime}$.

4. Let $\Gamma(P)$ be an original $F_{4}$-cycle associated with $P=\left\{p_{1}, \ldots, p_{k}\right\}$. Put $f P=\left\{q_{1}, \ldots, q_{k}\right\}$ where $q_{i}$ are singular planes associated with $p_{i}$. Then the map

$$
f_{p_{1}}^{\prime} \times \cdots \times f_{p_{k}}^{\prime}: W(P) \rightarrow W(f P)
$$

induces a map of $K$-cycles

$$
f_{p}: \Gamma(P) \rightarrow \Gamma(f P)
$$

as is easily seen.

Put $p_{i}=\left(\rho_{i}, n_{i}\right)$ and let $\rho_{i_{1}}, \ldots, \rho_{i_{r}}$ be short roots and the rest be long roots. Let $\theta_{i_{s}}^{\prime}, \theta_{i_{s}}^{\prime \prime}$ be the roots of $E_{6}$ associated with $\rho_{i_{s}}, 1 \leqq s \leqq r$. Further we put $q_{i_{s}}^{\prime}=\left(\theta_{i_{s}}^{\prime}, n_{i_{s}}\right)$ and $q_{i_{s}}^{\prime \prime}=\left(\theta_{i_{s}}^{\prime \prime}, n_{i_{s}}\right)$ for $1 \leqq s \leqq r$. Now consider an ordered set of singular planes

$$
Q=\left\{q_{1}, \ldots, q_{i_{1}-1}, q_{i_{1}}^{\prime}, q_{i_{1}}^{\prime \prime}, \ldots, q_{i_{s}}^{\prime}, q_{i_{s}}^{\prime \prime}, \ldots, q_{k}\right\}
$$

consisting of $k+r$ planes of codimension 1 in $R$. Then the product of maps

$$
\begin{array}{ll}
a_{j}: K\left(q_{j}\right) \rightarrow K\left(q_{j}\right) & \text { for } j \notin I, \\
b_{j}: K\left(q_{j}^{\prime}\right) \times K\left(q_{j}^{\prime \prime}\right) \rightarrow K\left(q_{j}^{\prime}\right) \cdot K\left(q_{j}^{\prime \prime}\right) \subset K\left(q_{j}\right) & \text { for } j \in I,
\end{array}
$$

where $I=\left\{i_{1}, \ldots, i_{r}\right\}, a_{j}$ are identity maps and $b_{j}$ are defined by the group multiplication in $E_{6}$, induces a homeomorphism

$$
\chi_{P}: \Gamma(Q) \approx \Gamma(f P) .
$$

If we identify $\Gamma(Q)$ and $I^{\prime}(f P)$ by $\psi_{P}$, then $H_{2}(\Gamma(f P) ; Z)$ has a homology basis consisting of sub $E_{6}$-cycles $K\left(q_{j}\right) / T, j \notin I$, and $K\left(q_{j}^{\prime}\right) / T, K\left(q_{j}^{\prime \prime}\right) / T, j \in I$. Its dual cohomology basis is written as $y_{j}, j \notin I, y_{j}^{\prime}, y_{j}^{\prime \prime}, j \in I$. Then

$$
\begin{aligned}
& f_{P}^{*}\left(y_{j}\right)=x_{j} \quad \text { for } j \notin I, \\
& f_{P}^{*}\left(y_{j}^{\prime}\right)=f_{P}^{*}\left(y_{j}^{\prime \prime}\right)=x_{j} \quad \text { for } j \in I
\end{aligned}
$$

by (8) and (10), where $\left\{x_{j}\right\}, 1 \leqq j \leqq k$, is the cohomology basis of $H^{2}(\Gamma(P) ; Z$ ) which is dual to the $F_{4}$-cycles $K\left(p_{i}\right) / T^{\prime}, 1 \leqq j \leqq k$. 
Let

$$
Q\left(i_{1}, \varepsilon_{1} ; i_{2}, \varepsilon_{2} ; \ldots ; i_{r}, \varepsilon_{r}\right) \quad\left(\varepsilon_{s}=1 \text { or } 2\right)
$$

be a subsequence of $Q$ which is obtained from $f P$ replacing $q_{i_{s}}$ by $q_{i_{s}}^{\prime}$ or by $q_{i_{s}}^{\prime \prime}$ according as $\varepsilon_{s}=1$ or 2 for $1 \leqq s \leqq r$. This defines a $2 k$-dimensional sub $E_{6}$-cycle of $\Gamma(f P)$ for each $\varepsilon_{1}, \ldots, \varepsilon_{r}$. Since $Q\left(i_{1}, \varepsilon_{1} ; i_{2}, \varepsilon_{2} ; \ldots ; i_{r}, \varepsilon_{r}\right)$ is the dual homology class of $y_{1} \cdots y_{i_{1}}^{\left(\varepsilon_{1}\right)} \cdots y_{i_{s}}^{\left(\varepsilon_{s}\right)} \cdots y_{k}$ by No. 1.3, (11) shows the

Proposition 6. The 2 k-cycles $f_{P} \Gamma(P)$ and $\sum \Gamma\left(Q\left(i_{1}, \varepsilon_{1} ; \ldots ; i_{r}, \varepsilon_{r}\right)\right)$ represent the same class in $H_{2 k}(\Gamma(f P) ; Z)$.

Let $\Omega f: \Omega F_{4} \rightarrow \Omega E_{6}$ be the map of loop spaces induced by $f$. Then we have the

PROPOSITION 7. $\Omega f_{*}\left(P_{*}\right)=\sum Q\left(i_{1}, \varepsilon_{1} ; \ldots ; i_{r}, \varepsilon_{r}\right)_{*}$ where $\Omega f_{*}$ denotes the homology map induced by $\Omega f$.

Let $c$ be a chain subject to $P$ in $R^{\prime}$. Then $\bar{f} c$ is a chain subject to $f P$ and to $Q\left(i_{1}, \varepsilon_{1} ; \ldots ; i_{r}, \varepsilon_{r}\right)$ in $R$, and we have a commutative diagram

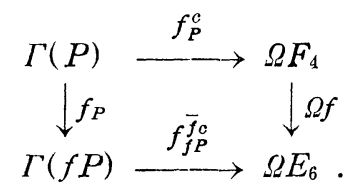

Now by the Prop. 6 we have immediately a proof of the Prop. 7 .

5. Now we discuss the homology map

$$
\Omega f_{*}: H_{*}\left(\Omega F_{4} ; Z\right) \rightarrow H_{*}\left(\Omega E_{6} ; Z\right)
$$

in deg. $\leqq 10$. Notations of Nos. 2.1 and 2.2 are used. If necessary to distinguish things associated with $F_{4}$ or with $E_{6}$ we denote them by attaching $\left(F_{4}\right)$ or $\left(E_{6}\right)$.

The homology bases of $H_{*}\left(\Omega F_{4} ; Z\right)$ and of $H_{*}\left(\Omega E_{6} ; Z\right)$ in deg. $\leqq 10$ are given in Nos. 2.1 and 2.2.

The planes $\left(\mu_{i}\left(E_{6}\right), 1\right)$ are associated with $\left(\mu_{i}\left(F_{4}\right), 1\right)$ for $0 \leqq i \leqq 2\left(\mu_{0}=\mu\right)$. The planes $\left(\mu_{i}\left(E_{6}\right), 1\right) \cap\left(\mu_{i}^{\prime}\left(E_{6}\right), 1\right)$ are associated with $\left(\mu_{i}\left(F_{4}\right), 1\right)$ for $i=3,4$. The plane $\left(\mu_{3}-\varphi_{3}^{\prime}\left(E_{6}\right), 1\right)$ is associated with $\left(\mu_{4}^{\prime}\left(F_{4}\right), 1\right)$. Then by the Prop. 7 we see that

$$
\begin{array}{ll}
\Omega f_{*}\left(P_{i *}\left(F_{4}\right)\right)=P_{i *}\left(E_{6}\right) & \text { for } 1 \leqq i \leqq 3, \\
\Omega f_{*}\left(P_{4 *}\left(F_{4}\right)\right)=P_{4 *}^{1}\left(E_{6}\right)+P_{4 *}^{2}\left(E_{6}\right), &
\end{array}
$$




$$
\begin{aligned}
\Omega f_{*}\left(P_{5 *}^{1}\left(F_{4}\right)\right) & =P_{5 *}^{1}\left(E_{6}\right)+P_{5 *}^{2}\left(E_{6}\right) \\
& +\left\{\left(\mu_{4}^{\prime}, 1\right), P_{4}^{1}\right\}_{*}+\left\{\left(\mu_{4}, 1\right), P_{4}^{2}\right\}_{*}, \\
\Omega f_{*}\left(P_{5 *}^{2}\left(F_{4}\right)\right) & =\left\{\left(\mu_{3}-\varphi_{3}^{\prime}, 1\right), P_{4}^{1}\right\}_{*}+\left\{\left(\mu_{3}-\varphi_{3}^{\prime}, 1\right), P_{4}^{2}\right\}_{*} .
\end{aligned}
$$

Now

$$
R_{\varphi_{4}^{\prime}}\left\{\left(\mu_{4}^{\prime}, 1\right), P_{4}^{1}\right\}=\left\{\left(\mu_{3}^{\prime}, 1\right), P_{4}^{1}\right\}=P_{5}^{3}\left(E_{6}\right) .
$$

Hence by the Prop. 2

$$
\left\{\left(\mu_{4}^{\prime}, 1\right), P_{4}^{1}\right\}_{*}=P_{5 *}^{3}\left(E_{6}\right) .
$$

Similarly

$$
\left\{\left(\mu_{4}, 1\right), P_{4}^{2}\right\}_{*}=P_{5 *}^{4}=P_{5 *}^{3}\left(E_{6}\right) .
$$

Therefore

$$
\Omega f_{*}\left(P_{5 *}^{1}\left(F_{4}\right)\right)=P_{5 *}^{1}\left(E_{6}\right)+P_{5 *}^{2}\left(E_{6}\right)+2 P_{5 *}^{3}\left(E_{6}\right) .
$$

Next, $\mu_{3}-\varphi_{3}^{\prime}=\mu_{3}^{\prime}-\varphi_{3}$. Then by the Prop. 1

$$
\left\{\left(\mu_{3}-\varphi_{3}^{\prime}, 1\right), P_{4}^{1}\right\}_{*}=P_{5 *}^{3}-\left\{\left(\varphi_{3}, 0\right), P_{4}^{1}\right\}_{*} .
$$

Put $q=\left(\varphi_{3}, 0\right) \cap\left(\mu_{3}, 1\right)$. Since $\varphi_{3}+\mu_{3}=\mu_{2},\left(\mu_{2}, 1\right)$ contains $q$ and the semisimple part of $K(q)$ is of type $A_{2}$. Put $P^{\prime}=\left\{\left(\varphi_{3}, 0\right), P_{4}^{1}\right\}$ and $P^{\prime \prime}=\left\{q,\left(\mu_{1}, 1\right),(\mu, 1)\right\}$, and let

$$
\alpha: K\left(\varphi_{3}\right) \times K\left(\mu_{3}\right) \times K\left(\mu_{2}\right) \rightarrow K\left(\varphi_{3}\right) \cdot K\left(\mu_{3}\right) \cdot K\left(\mu_{2}\right) \subset K(q)
$$

be defined by the group multiplication.

$$
\alpha \times \bullet \times \iota: W\left(P^{\prime}\right) \rightarrow W\left(P^{\prime \prime}\right)
$$

induces

$$
\ddot{\alpha}: \Gamma\left(P^{\prime}\right) \rightarrow \Gamma\left(P^{\prime \prime}\right)
$$

where $c$ is identity map.

Let $c$ be a chain in $R$ subject to $P^{\prime \prime}$. $c$ is considered also as a chain subject to $P^{\prime}$ with the second and third polygons collapsed to a point. The diagram

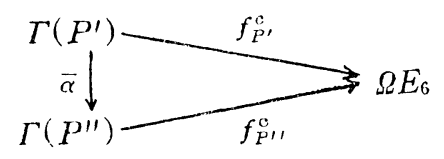

is clearly commutative. Then, since $\Gamma^{\prime}\left(P^{\prime}\right)$ and $\Gamma\left(P^{\prime \prime}\right)$ have the same dimension we see that

$$
P_{*}^{\prime}=\beta P_{*}^{\prime \prime} \quad \text { with } \beta \in Z \text {. }
$$


On the other hand, by the Prop. $3 P_{*}^{\prime \prime}=0$, whence $P_{*}^{\prime}=0$. Consequently

Similarly

$$
\left\{\left(\mu_{3}-\varphi_{3}^{\prime}, 1\right), P_{4}^{1}\right\}_{*}=P_{5 *}^{3}\left(E_{6}\right) \text {. }
$$

$$
\left\{\left(\mu_{3}-\varphi_{3}^{\prime}, 1\right), P_{4}^{2}\right\}_{*}=P_{5 *}^{4}=P_{5 *}^{3}\left(E_{6}\right)
$$

Therefore

$$
\Omega_{*}\left(P_{5 *}^{2}\left(F_{4}\right)\right)=2 P_{5 *}^{3}\left(E_{6}^{\prime}\right) .
$$

(12), (13) and (14) describe the integral homology map $\Omega f_{*}$ in deg. $\leqq 10$. From these we see easily that

Profosition 8. The homology map $\Omega f_{*} \bmod p$ is injective in deg. $\leqq 10$ for any odd prime $p$.

Proposition 8'. The homology map $\Omega f_{*} \bmod 2$ is injective in deg. $\leqq 8$. $\Omega f_{*} \mid H_{10}\left(\Omega F_{4} ; Z_{2}\right)$ has the kernel of dimension 1 (generated by $P_{5 *}^{2}\left(F_{4}\right)$ ).

§. The homology map $\boldsymbol{H}_{*}\left(\Omega E_{6}\right) \rightarrow \boldsymbol{H}_{*}\left(\Omega E_{7}\right)$

1. In the Schläfli figure of No. 2.3, the centralizer of the straight line $\bigcap_{i=2}^{7}\left(\varphi_{i}, 0\right)$ is of type $E_{6} \times T_{1},[7]$. As the semisimple part of this subgroup we obtain the canonical inclusion $E_{6} \subset E_{7}$. In this inclusion the dominant root of $E_{0}$ is not obtained as the restriction of the dominant root of $E_{\tau}$ to $E_{0} \cap T$ ( $T$ is the maximal torus of $E_{i}$ ). By this reason we use another fundamental system of roots described by the following figure.

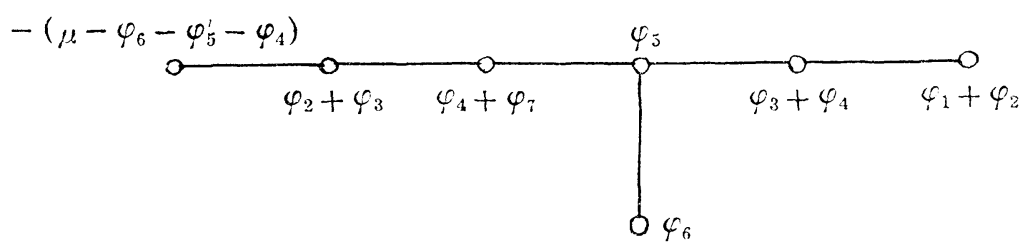

Then the inclusion $g: E_{6} \subset E_{7}$ is defined as the subgroup generated by the closed system of roots spanned by $\left(\varphi_{2}+\varphi_{3}, \varphi_{4}+\varphi_{7}, \varphi_{5}, \varphi_{3}+\varphi_{4}, \varphi_{1}+\varphi_{2}, \varphi_{0}\right)$. This is equivalent to the canonical inclusion. Hence its homological effects are the same with the effects of the canonical inclusion.

2. Let $T$ be the maximal torus of $E_{\bar{\tau}}, T^{\prime}=E_{0} \cap T$ be the maximal torus of $E_{\tilde{0}}$ and $g: R^{\prime} \subset R$ be the induced inclusion of the universal covering groups as in $\$ 3$.

For each singular plane $p=(\rho, n)$ in $R^{\prime}$, let $q=(\theta, n)$ be the associated plane in $R$ in the sense that $p=R^{\prime} \cap q$. Then clearly $g(K(p)) \subset K(q)$ and the 
induced homeomorphism $g_{p}: K(p) / T^{\prime} \approx K(q) / T$ is orientation preserving.

Let $\Gamma(P)$ be an original $E_{\hat{0}}$-cycle with $P=\left\{p_{1}, \ldots, p_{k}\right\}$. Put $g P=\left\{q_{1}, \ldots\right.$, $q_{k}$ \} where $q_{i}$ singular planes in $R$ associated with $p_{i} . \quad \Gamma(g P)$ is an original $E_{\gamma^{-}}$ cycle. The map

$$
g_{p_{1}}^{\prime} \times \cdots \times g_{p_{k}}^{\prime}: W(P) \rightarrow W(g P)
$$

induces a homeomorphism of $K$-cycles

$$
g_{P}: \Gamma(P) \approx \Gamma(g P)
$$

preserving orientations. Let

$$
\Omega g: \Omega E_{6} \rightarrow \Omega E_{7}
$$

be the map of loop spaces induced by $g$. Then it is immediate to see that

$$
\Omega g_{*}\left(P_{*}\right)=(g P)_{*}
$$

where $\Omega g_{*}$ is the map of integral homology groups induced by $\Omega g$.

3. We discuss the homology map

$$
\Omega g_{*}: H_{*}\left(\Omega E_{6} ; Z\right) \rightarrow H_{*}\left(\Omega E_{7} ; Z\right)
$$

in deg. $\leqq 10$. Notations of Nos. 2.2 and 2.3 are used.

The planes $\left(\mu_{i}\left(E_{7}\right), 1\right)$ are associated with $\left(\mu_{i}\left(E_{6}\right), 1\right)$ for $0 \leqq i \leqq 2\left(\mu_{0}=\mu\right)$. The planes $\left(\mu_{4}^{\prime}\left(E_{i}\right), 1\right),\left(\mu_{4}\left(E_{\tau}\right), 1\right),\left(\mu_{5}-\varphi_{i}\left(E_{i}\right), 1\right)$ and $\left(\mu_{i}\left(E_{i}\right), 1\right)$ are associated with $\left(\mu_{3}\left(E_{\hat{0}}\right), 1\right),\left(\mu_{3}^{\prime}\left(E_{6}\right), 1\right),\left(\mu_{4}\left(E_{6}\right), 1\right)$ and $\left(\mu_{4}^{\prime}\left(E_{6}\right), 1\right)$ respectively.

Then by (15) we see that

$$
\begin{aligned}
& \Omega g_{*}\left(P_{i *}\left(E_{6}^{\prime}\right)\right)=P_{i *}\left(E_{7}\right) \quad \text { for } 1 \leqq i \leqq 3, \\
& \Omega g_{*}\left(P_{4 *}^{\prime}\left(E_{6}\right)\right)=\left\{\left(\mu_{4}^{\prime}, 1\right), P_{3}\left(E_{7}\right)\right\}_{*}, \\
& \Omega g_{*}\left(P_{4 *}^{2}\left(E_{6}\right)\right)=\left\{\left(\mu_{4}, 1\right), P_{3}\left(E_{7}\right)\right\}_{*}, \\
& \Omega g_{*}\left(P_{5 *}^{1}\left(E_{6}\right)\right)=\left\{\left(\mu_{5}-\varphi_{7}, 1\right),\left(\mu_{4}^{\prime}, 1\right), P_{3}\left(E_{7}\right)\right\}_{*}, \\
& \Omega g_{*}\left(P_{5 *}^{2}\left(E_{6}\right)\right)=\left\{\left(\mu_{6}, 1\right),\left(\mu_{4}, 1\right), P_{3}\left(E_{7}\right)\right\}_{*}, \\
& \Omega g_{*}\left(P_{5 *}^{3}\left(E_{6}\right)\right)=\left\{\left(\mu_{4}, 1\right),\left(\mu_{4}^{\prime}, 1\right), P_{3}\left(E_{7}\right)\right\}_{*} .
\end{aligned}
$$$$
\left(16^{\prime \prime} .\right. \text { iii ) }
$$

Now

$$
R_{\vartheta_{7}}\left\{\left(\mu_{4}^{\prime}, 1\right), P_{3}\right\}=P_{4}\left(E_{7}\right), R_{\rho_{3}}\left\{\left(\mu_{4}, 1\right), P_{3}\right\}=P_{4}\left(E_{7}\right),
$$

where $R_{f_{i}}$ denotes the reflection across the plane $\left(\varphi_{i}, 0\right)$. Hence by the Prop. 2 and $\left(16^{\prime} . \mathrm{i}\right)$ and $\left(16^{\prime} . \mathrm{ii}\right)$ we see that 


$$
\Omega g_{*}\left(P_{4 *}^{i}\left(E_{\tilde{6}}\right)\right)=P_{4 *}\left(E_{\tau}\right) \quad \text { for } i=1,2
$$

Next

$$
\begin{aligned}
& R_{\gamma_{2}} R_{p_{7}}\left\{\left(\mu_{5}-\varphi_{i}, 1\right),\left(\mu_{4}^{\prime}, 1\right), P_{3}\right\}=P_{5}^{1}\left(E_{7}\right), \\
& R_{\gamma_{2}} R_{\gamma_{1}} R_{\gamma_{3}}\left\{\left(\mu_{0}, 1\right),\left(\mu_{4}, 1\right), P_{3}\right\}=P_{5}^{1}\left(E_{7}\right), \\
& R_{\gamma}\left\{\left(\mu_{4}, 1\right),\left(\mu_{4}^{\prime}, 1\right), P_{3}\right\}=\left\{\left(\mu_{5}^{\prime}, 1\right), P_{4}\left(E_{7}\right)\right\} .
\end{aligned}
$$

Since $\mu_{5}^{\prime}+\varphi_{3}=\mu_{1}^{\prime}$, by the Prop. 1 we have

$$
\left\{\left(\mu_{5}^{\prime}, 1\right), P_{4}\left(E_{7}\right)\right\}_{*}=P_{5 *}^{2}\left(E_{7}\right)-\left\{\left(\varphi_{3}, 0\right), P_{4}\left(E_{7}\right)\right\}_{*} .
$$

Then, applying the same argument with the proof of (14) (use the Prop. 3 for $\left.q=\left(\varphi_{3}, 0\right) \cap\left(\mu_{3}, 1\right) \cap\left(\mu_{3}, 1\right)\right)$, we see that

$$
\left\{\left(\varphi_{3}, 0\right), P_{4}\left(E_{7}\right)\right\}_{+}=0 \text {. }
$$

Hence by the Prop. 2 and (16".i), ii) and iii) we have the

$$
\begin{aligned}
& \Omega g_{*}\left(P_{5 *}^{i}\left(E_{6}\right)\right)=P_{5 *}^{1}\left(E_{7}\right) \quad \text { for } i=1,2, \\
& \Omega g_{*}\left(P_{5 *}^{3}\left(E_{6}\right)\right)=P_{5 *}^{2}\left(E_{7}\right) .
\end{aligned}
$$

(16)-(18) describe the integral homology map $\Omega g_{*}$ in deg. $\leqq 10$. From these we see immediately the

Proposition 9. The homology map $\Omega g_{*} \bmod p$ is surjective in deg. $\leqq 10$ for any prime $p$.

$\S 5$. The homology map $H_{*}\left(\Omega E_{7}\right) \rightarrow H_{*}\left(\Omega E_{8}\right)$

1. By the same reason with $\S 4$ we use the different fundamental system of roots described by the following figure to define the inclusion $h: E_{\tau} \subset E_{8}$.

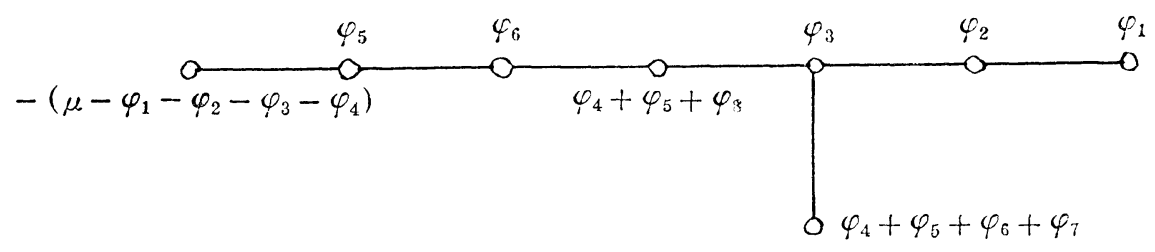

The inclusion $h$ is defined as the subgroup generated by the closed system of roots spanned by $\left(\varphi_{5}, \varphi_{6}, \varphi_{4}+\varphi_{5}+\varphi_{8}, \varphi_{3}, \varphi_{2}, \varphi_{1}, \varphi_{4}+\varphi_{5}+\varphi_{6}+\varphi_{7}\right)$. This is equivalent to the canonical inclusion and its homological effects are the same with those of the canonical one.

Let $T$ be the maximal torus of $E_{3}, T^{\prime}=T \cap E_{i}$ be the maximal torus of $E_{\bar{\gamma}}$ and $\bar{h}: R^{\prime} \subset R$ be the inclusion of the universal covering groups of $T^{\prime}$ and $T$.

With each singular plane $p=(\rho, n)$ in $R^{\prime}$ there is associated a singular 
plane $q=(\theta, n)$ in $R$ in the sense that $p=R^{\prime} \cap q$. For each ordered set $P=\left\{p_{1}\right.$, $\left.\ldots, p_{k}\right\}$ of singular planes with codim $p_{i}=1$ we define $h P=\left\{q_{1}, \ldots, q_{k}\right\}$ such that $q_{i}$ are associated with $p_{i}$ for $1 \leqq i \leqq k$. Let

$$
\Omega h: \Omega E_{7} \rightarrow \Omega E_{8}
$$

be the map of loop spaces induced by $h$ and $\Omega h_{*}$ be the map of integral homology groups induced by $\Omega h$. Then, in the same way with No. 4.2 we see that

$$
\Omega h_{*}\left(P_{\psi}\right)=(h P)_{\psi} .
$$

2. We discuss the homology map

$$
\Omega h_{;}: H_{*}\left(\Omega E_{7} ; Z\right) \rightarrow H_{*}\left(\Omega E_{8} ; Z\right)
$$

in deg. $\leqq 14$. Notations of Nos. 2.3 and 2.4 are used.

The planes $\left(\mu_{i}\left(E_{8}\right), 1\right)$ are associated with $\left(\mu_{i}\left(E_{7}\right), 1\right)$ for $0 \leqq i \leqq 3\left(\mu_{0}=\mu\right)$. The planes $\left(\mu_{6}^{\prime}\left(E_{8}\right), 1\right),\left(\mu_{5}-\varphi_{8}\left(E_{8}\right), 1\right)$ and $\left(\mu-\varphi_{8}-\varphi_{5}\left(E_{3}\right), 1\right)$ are associated with $\left(\mu_{i}\left(E_{7}\right), 1\right)$ for $4 \leqq i \leqq 6$ in its order, and $\left(\mu_{0}-\varphi_{7}\left(E_{8}\right), 1\right)$ and $\left(\mu_{6}-\varphi_{7}-\varphi_{8}\right.$ $\left.-\varphi_{5}-\varphi_{4}\left(E_{8}\right), 1\right)$ are associated with $\left(\mu_{1}^{\prime}\left(E_{7}\right), 1\right)$ and $\left(\mu_{5}^{\prime}\left(E_{7}\right), 1\right)$ respectively.

Then by (19) we see that

$$
\Omega h_{*}\left(P_{i *}\left(E_{\tau}\right)\right)=P_{i *}\left(E_{\delta}\right) \quad \text { for } 1 \leqq i \leqq 4,
$$

$$
\Omega h_{*}\left(P_{5 *}^{1}\left(E_{7}\right)\right)=\left\{\left(\mu_{6}^{\prime}, 1\right), P_{4}\left(E_{8}\right)\right\}_{*},
$$

$$
\Omega h_{*}\left(P_{5 *}^{2}\left(E_{7}\right)\right)=\left\{\left(\mu_{6}-\varphi_{7}, 1\right), P_{4}\left(E_{8}\right)\right\}_{*},
$$

$$
\Omega h_{*}\left(P_{6 *}^{1}\left(E_{7}\right)\right)=\left\{\left(\mu_{6}-\varphi_{8}, 1\right),\left(\mu_{6}^{\prime}, 1\right), P_{4}\right\}_{*},
$$

$$
\Omega h_{*}\left(P_{6 *}^{2}\left(E_{7}\right)\right)=\left\{\left(\mu_{0}-\varphi_{7}, 1\right),\left(\mu_{6}^{\prime}, 1\right), P_{4}\right\}_{*},
$$

$$
\begin{aligned}
& \Omega h_{*}\left(P_{7 *}^{1}\left(E_{7}\right)\right)=\left\{\left(\mu_{6}-\varphi_{8}-\varphi_{5}, 1\right),\left(\mu_{0}-\varphi_{8}, 1\right),\left(\mu_{6}^{\prime}, 1\right), P_{4}\right\}_{*}, \\
& \Omega h_{*}\left(P_{7 *}^{2}\left(E_{7}\right)\right)=\left\{\left(\mu_{0}-\varphi_{7}, 1\right),\left(\mu_{6}-\varphi_{3}, 1\right),\left(\mu_{6}^{\prime}, 1\right), P_{4}\right\}_{*} .
\end{aligned}
$$

Firstly

$$
R_{\varphi 5} R_{\vartheta_{3}}\left\{\left(\mu_{6}^{\prime}, 1\right), P_{4}\right\}=P_{5}\left(E_{8}\right), R_{\% 5} R_{\gamma_{6}} R_{\varphi_{s}}\left\{\left(\mu_{6}-\varphi_{7}, 1\right), P_{4}\right\}=P_{5}\left(E_{8}\right) .
$$

Hence by the Prop. 2 and $\left(20^{\prime} . \mathrm{i}\right)$ and ii) we see that

$$
2 h_{k}\left(P_{5 *}^{i}\left(E_{7}\right)\right)=P_{5 *}\left(E_{8}\right) \quad \text { for } i=1,2 .
$$

Secondly

$$
\begin{aligned}
& R_{p 6} R_{p 8} R_{p_{3}}\left\{\left(\mu_{0}-\varphi_{8}, 1\right),\left(\mu_{6}^{\prime}, 1\right), P_{4}\right\}=P_{6}\left(E_{8}\right), \\
& R_{p 8} R_{p 8} R_{p_{7}}\left\{\left(\mu_{6}-\varphi_{7}, 1\right),\left(\mu_{6}^{\prime}, 1\right), P_{4}\right\}=\left\{\left(\mu_{0}-\varphi_{3}-\varphi_{5}, 1\right), P_{5}\left(E_{8}\right)\right\} .
\end{aligned}
$$


Since $\mu_{6}-\varphi_{8}-\varphi_{5}+\left(\varphi_{5}+\varphi_{6}+\varphi_{8}\right)=\mu_{5}$, by the Prop. 1

$$
\left\{\left(\mu_{6}-\varphi_{3}-\varphi_{5}, 1\right), P_{5}\right\}_{*}=P_{0 *}-\left\{\left(\varphi_{5}+\varphi_{6}+\varphi_{3}, 0\right), P_{5}\right\}_{*} .
$$

Further $R_{\hat{\gamma} 8} R_{\hat{\gamma}}\left\{\left(\varphi_{5}+\varphi_{\hat{0}}+\varphi_{8}, 0\right), P_{5}\right\}=\left\{\left(\varphi_{5}, 0\right), P_{5}\right\}$,

$$
\begin{aligned}
\left\{\left(\varphi_{5}, 0\right), P_{5}\right\}_{*} & =-P_{6 *}+\left\{\left(\mu_{1}, 1\right),\left(\mu_{4}, 1\right), P_{4}\right\}_{*} & & \text { by the Prop. } 1, \\
& =-P_{6 *} & & \text { by the Prop. } 5 .
\end{aligned}
$$

Therefore by the Prop. 2 and $\left(20^{\prime \prime}\right.$. i) and ii) we see the

$$
\begin{aligned}
& \Omega h_{*}\left(P_{6 *}^{1}\left(E_{7}\right)\right)=P_{6 *}\left(E_{3}\right), \\
& \Omega h_{*}\left(P_{6 *}^{2}\left(E_{7}\right)\right)=2 P_{6 *}\left(E_{5}\right) .
\end{aligned}
$$

Thirdly

$$
\begin{gathered}
R_{\vartheta_{6}} R_{\% 5} R_{p 8}\left\{\left(\mu_{6}-\varphi_{8}-\varphi_{5}, 1\right),\left(\mu_{6}-\varphi_{8}, 1\right),\left(\mu_{6}^{\prime}, 1\right), P_{4}\right\}=P_{7}^{2}, \\
R_{\vartheta_{6}} R_{\sigma_{5}} R_{98}\left\{\left(\mu_{6}-\varphi_{7}, 1\right),\left(\mu_{6}-\varphi_{8}, 1\right),\left(\mu_{6}^{\prime}, 1\right), P_{4}\right\} \\
=\left\{\left(\mu_{6}-\varphi_{7}-\varphi_{8}-\varphi_{5}-\varphi_{6}, 1\right), P_{6}\right\} .
\end{gathered}
$$

By the Prop. 1 we have

$$
\left.\left\{\left(\mu_{0}-\varphi_{7}-\varphi_{8}-\varphi_{5}-\varphi_{6}, 1\right), P_{0}\right\}_{*}=P_{7 *}^{1}-\left\{\varphi_{5}+\varphi_{0}+\varphi_{7}+\varphi_{s}, 0\right), P_{0}\right\}_{*} .
$$

Further

$$
R_{\sigma_{7}}\left\{\left(\varphi_{5}+\varphi_{6}+\varphi_{7}+\varphi_{8}, 0\right), P_{6}\right\}=\left\{\left(\varphi_{5}+\varphi_{6}+\varphi_{8}, 0\right), P_{6}\right\}
$$

and

$$
\left\{\left(\varphi_{5}+\varphi_{0}+\varphi_{8}, 0\right), P_{6}\right\}_{*}=\left\{\left(\varphi_{5}, 0\right), P_{0}\right\}_{*}+\left\{\left(\varphi_{6}, 0\right), P_{0}\right\}_{*}+\left\{\left(\varphi_{8}, 0\right), P_{6}\right\}_{*}
$$

by the Prop. 1 . Here

$$
\left\{\left(\varphi_{5}, 0\right), P_{6}\right\}_{*}=0
$$

by an argument used in the proof of (14) (for $q=\left(\varphi_{5}, 0\right) \cap\left(\mu_{5}, 0\right) \cap\left(\mu_{4}, 0\right)$ ), and

$$
\left\{\left(\varphi_{6}, 0\right), P_{6}\right\}_{*}=-P_{7 *}^{1},\left\{\left(\varphi_{8}, 0\right), P_{\dot{0}}\right\}_{*}=-P_{7 *}^{2}
$$

by an argument used in the proof of (21). Hence by the Prop. 2 and (20'" . i) and ii) we see that

$$
\begin{aligned}
& \Omega h_{*}\left(P_{7 *}^{1}\left(E_{7}\right)\right)=P_{7 *}^{2}\left(E_{8}\right), \\
& \Omega h_{*}\left(P_{7 * *}^{2}\left(E_{7}\right)\right)=2 P_{7 *}^{1}+P_{7 *}^{2}\left(E_{s}\right) .
\end{aligned}
$$

(20)-(22) describe the integral homology map $\Omega h_{*}$ in deg. $\leqq 12$ completely, whereas (23) describes $\Omega h_{*}$ in deg. 14 partly with the exception of $\Omega h_{*}\left(P_{7 *}^{3}\left(E_{i}\right)\right)$. Nevertheless by $(20)-(23)$ we see immediately the following 
Proposition 10. The homology map $\Omega g_{*} \bmod p$ is surjective in deg. $\leqq 14$ for any odd prime p.

\section{Chapter III. The cohomology maps mod 3 induced by the inclusions $F_{4} \subset E_{6} \subset E_{7} \subset E_{8}$}

\section{§ 1. Preliminaries}

1. By $K$ we denote any one of groups $F_{4}, E_{0}, E_{7}, E_{8}$ when they are discussed at the same time. Similarly by $k$ we denote any one of inclusions $f, g, h$.

$H^{*}\left(\Omega K ; Z_{3}\right)$ in low degrees are described as follows :

$$
\begin{aligned}
& H^{*}\left(\Omega F_{4} ; Z_{3}\right)=Z_{3}\left[u_{2}, u_{10}\right] \text { in deg. } \leqq 10, \\
& H^{*}\left(\Omega E_{0} ; Z_{3}\right)=Z_{3}\left[u_{2}, u_{8}, u_{10}\right] \text { in deg. } \leqq 10, \\
& H^{*}\left(\Omega E_{7} ; Z_{3}\right)=Z_{3}\left[u_{2}, u_{10}, u_{14}\right]: \text { in deg. } \leqq 14, \\
& H^{*}\left(\Omega E_{8} ; Z_{3}\right)=Z_{3}\left[u_{2}, u_{14}\right] \text { in deg. } \leqq 14,
\end{aligned}
$$

where suffixes of generators denote degrees. $u_{2}$ is defined as the dual class of $P_{1 *}$. Then, since $\Omega k_{*}\left(P_{1 *}\right)=P_{1 *}$ by (II.12), (II.16) and (II.20) we see that

$$
\Omega k^{*}\left(u_{2}\right)=u_{2}
$$

where $\Omega k^{*}$ denotes the cohomology map mod 3 induced by $\Omega k$. By a discussion of Bott-Samelson [10], Chap. II, §13, we know that

$$
u_{2}^{3} \neq 0
$$

for $K=E_{\hat{0}}, E_{\tau}$ and $E_{3}$. Since $\Omega f^{*} \mid H^{6}\left(\Omega E_{0} ; Z_{3}\right)$ is bijective by (II. 12), we see that (3) is true also for $K=F_{4}$. By (3) and the Poincaré polynomials of compact exceptional groups (e.g., cf. [8]), we can see that (1) is true.

2. $H^{*}\left(K ; Z_{3}\right)$ are known by $[3,5,6]$. All elements of them have height $\leqq 3$ and their systems of generators of type $(M)$ are as follows:

$$
\begin{aligned}
& F_{4}: x_{3}, x_{7}, x_{8}, x_{11}, x_{15} ; \\
& E_{6}: x_{3}, x_{7}, x_{8}, x_{9}, x_{11}, x_{15}, x_{17} ; \\
& E_{7}: x_{3}, x_{7}, x_{3}, x_{11}, x_{15}, \ldots ; \\
& E_{8}: x_{3}, x_{7}, x_{3}, x_{15}, \ldots .
\end{aligned}
$$

The omitted generators are not needed in our present discussion. The following relations about Steenrod reduced powers and Bockstein operation hold:

$$
x_{\bar{\tau}}=\mathscr{P}^{1} x_{3}, \quad x_{\mathrm{S}}=\delta_{3}^{*} x_{\bar{\tau}} \quad \text { for all groups } K,
$$




$$
x_{15}=\mathscr{P}^{1} x_{11} \quad \text { for } K=F_{4}
$$

3. In the spectral sequences mod 3 associated with usual fibrations of loop spaces of $K$ ( $K=$ base space, $\Omega K=$ the fibre), the generators of fibre cohomologies described in (1) are all transgressive and the transgression image of $u_{i}$ is represented by $x_{i+1}$.

This is proved by discussing these spectral sequences one by one, and of course not generally true. For example, $H^{*}\left(\Omega G_{2} ; Z_{3}\right)$ has generators $u_{2}, u_{6}$, $\ldots$, with $u_{2}^{3}=0$, and the second generator $u_{0}$ is not transgressive.

The transgression image of $u_{2}$ is the well-defined element $x_{3}$. Hence, by (2) we see that $k^{*}\left(x_{3}\right)=x_{3}$. Consequently, by (5) we see that

$$
k^{*}\left(x_{i}\right)=x_{i} \quad \text { for } i=3,7,8 \text {. }
$$

The generators $x_{3}, x_{7}$ and $x_{5}$ are universally transgressive. Hence, by making use of Theorem WCSS of Chap. I we see that

$$
H^{*}\left(B_{K} ; Z_{3}\right)=Z_{3}\left[y_{4}, y\right] \otimes \Lambda_{3}\left(y_{9}\right) \quad \text { in deg. } \leqq 9
$$

for all $K=F_{4}, E_{6}, E_{7}$ and $E_{8}$, where $y_{4}$ is the image of $x_{3}$ by the universal transgression and $y_{8}=\mathscr{P}^{1} y_{4}, y_{9}=\delta_{3}^{*} y_{8}$.

§2. $\boldsymbol{f}^{*}: \boldsymbol{H}^{*}\left(\boldsymbol{E}_{6} ; \boldsymbol{Z}_{3}\right) \rightarrow \boldsymbol{H} *\left(\boldsymbol{F}_{4} ; \boldsymbol{Z}_{3}\right)$

1. We choose the generators of $H^{*}\left(F_{4} ; Z_{3}\right)$ to satisfy the Prop. I.2. Consider the spectral sequences mod 3 of loop spaces of $F_{4}$ and $E_{\hat{0}}$, and the homomorphism of them induced by $f$.

$\Omega f^{*}$-image of $u_{10}$ of $\Omega E_{0}$ must be transgressive. If $\Omega f^{*}\left(u_{10}\right)=0$, then $u_{10}$ of $\Omega F_{4}$ is not in the images of $\Omega f^{*}$. This contradicts the Prop. II. 8 because we see that $\Omega f^{*}$ is surjective in degree 10 by this proposition. Hence we can choose $u_{10}$ of $\Omega E_{\hat{0}}$ such that

$$
\Omega f^{*}\left(u_{10}\right)=u_{10} .
$$

Then, by No. 1.3 , we see that $f^{*}\left(x_{11}\right)=x_{11}+a x_{3} x_{9}$ with $a \in Z_{3}$.

We can choose $x_{11}$ of $E_{6}$ such that it is $d_{r}$-cocycles for $r \leqq 4$ in the universal spectral sequence. Then, using the Prop. I. 2 we see that

$$
f^{*}\left(x_{11}\right)=x_{11}
$$

Now, by $\left(5^{\prime}\right) f^{*}\left(\mathscr{P}^{1} x_{11}\right)=x_{15}$ is indecomposable in $H^{*}\left(F_{4} ; Z_{3}\right)$. Hence $\mathscr{P}^{1} x_{11}$ is not decomposable in $H^{*}\left(F_{6}: Z_{3}\right)$, and we can choose the generator $x_{15}$ of 
$H^{*}\left(E_{6} ; Z_{3}\right)$ to satisfy

$$
x_{15}=\mathscr{P}^{1} x_{11} .
$$

Then $f^{*} x_{15}=x_{15}$, and by (4), (7) and (7') we see that $f^{*} \bmod 3$ is surjective. Hence we have the

Proposition 1. $F_{4}$ is totally non homologous zero mod 3 in $E_{6}$.

2. Proposition 2. In the universal spectral sequence mod 3 of $E_{0}$ the behaviors of generators of $H^{*}\left(E_{6} ; Z_{3}\right)$ except $x_{17}$ are as follows: $x_{3}, x_{7}, x_{8}$ and $x_{9}$ are universally transgressive; $x_{11}$ and $x_{15}$ are never universally transgressive and they can be chosen such that

$$
\begin{aligned}
& d_{i} \kappa_{i}^{2} x_{11}=d_{i} \kappa_{i}^{2} x_{i 5}=0 \quad \text { for } 2 \leqq i<9, \\
& d_{3} \kappa_{9}^{2} x_{11}=\kappa_{9}^{2}\left(y_{3} \otimes x_{3}\right) \neq 0, \\
& d_{3} \kappa_{9}^{2} x_{15}=\kappa_{9}^{2}\left(y_{3} \otimes x_{7}\right) \neq 0 .
\end{aligned}
$$

The fact that $x_{9}$ can be chosen to be universally transgressive is easily seen. Then the Prop. I. $2,\left(6^{\prime}\right)$ and $\left(7^{\prime}\right)$ prove this proposition.

Corollary. $H^{*}\left(B_{F^{\prime}} ; Z_{3}\right)=Z_{3}\left[y_{1}, y_{5}, y_{10}\right] \otimes A_{3}\left(y_{9}\right) /\left(y_{1} y_{9}, y_{5} y_{5}\right)$ in deg. $\leqq 17$ where $y_{10} \in H^{10}\left(B_{F_{6}} ; Z_{3}\right)$ is a representative of the transgression image of $x_{9}$.

Remark. The behavior of $x_{17}$ in the universal spectral sequence is not determined. About this we have two possibilities: $x_{17}$ can be chosen to be universally transgressive or not. If $x_{17}$ is never universally transgressive, then we can choose it such that $d_{i} \kappa_{i}^{2} x_{1 i}=0$ for $2 \leqq i<10$ and $d_{10} \kappa_{10}^{2} x_{17}=\kappa_{10}^{2}\left(y_{10} \otimes x_{8}\right) \neq 0$.

3. The coproduct $\psi^{*}: H^{*}\left(E_{0} ; Z_{3}\right) \rightarrow H^{*}\left(E_{0} ; Z_{3}\right) \otimes H^{\dagger}\left(E_{6} ; Z_{3}\right)$ is determined in the same way as Theorem I. 1 .

Proposition 3. For generators of $H^{*}\left(E_{6} ; Z_{3}\right)$ which behave in the universal spectral sequence as stated in the Prop. 2 (and the remark to it) their coproducts are as follows:
a) $x_{3}, x_{i}, x_{3}$ and $x_{9}$ are primitive,
b) $\psi^{*} x_{11}=1 \otimes x_{11}+x_{11} \otimes 1+x_{8} \otimes x_{3}$,
c) $\psi^{*} x_{15}=1 \otimes x_{15}+x_{15} \otimes 1+x_{3} \otimes x_{i}$,
d) $\psi^{*} x_{17}=1 \otimes x_{17}+x_{17} \otimes 1+\varepsilon x_{3} \otimes x_{s}, \quad \varepsilon \in Z_{3}$,

where $\varepsilon=0$ or 1 according as $x_{17}$ can be chosen to be universally transgressive 
or not.

Then the Pontrjagin ring $H_{*}\left(E_{0} ; Z_{3}\right)$ can be discussed in the same way as Theorem I.2.

Theorem 1. The Pontrjagin ring $H_{*}\left(E_{5} ; Z_{3}\right)$ is non-commutative and has a 3-simple system of generators $v_{3}, v_{7}, v_{3}, v_{9}, v_{11}, v_{15}$ and $v_{17}$ satisfying the following relations

$$
\begin{aligned}
& v_{i} \vee v_{i}=0 \text { for } i \neq 8, v_{s} \vee v_{s} \vee v_{s}=0 \\
& {\left[v_{i}, v_{j}\right]=0 \text { for } i<j \text { and }(i, j) \neq(3,8),(7,8),(8,9)} \\
& {\left[v_{s}, v_{3}\right]=v_{11},\left[v_{3}, v_{i}\right]=v_{15},\left[v_{y}, v_{8}\right]=\varepsilon v_{17}}
\end{aligned}
$$

where $\left[v_{i}, v_{j}\right]=v_{i} \vee v_{j}-(-1)^{i j} v_{j} \vee v_{j}$ and $\varepsilon$ is the same with that in the Prop. 3.

Remark. The generators $v_{i}, i=3,7,8,11,15$ of $H_{*}\left(E_{0} ; Z_{3}\right)$ can be chosen as the $f_{*}$-images of generators $v_{i}$ of $H_{*}\left(F_{4} ; Z_{3}\right)$. Then the relations of noncommutativity $\left[v_{s}, v_{3}\right]=v_{11},\left[v_{s}, v_{7}\right]=v_{15}$ are inherited from the corresponding ones in $H^{*}\left(F_{4} ; Z_{3}\right)$.

4. $H^{*}\left(\operatorname{Ad} E_{0} ; Z_{3}\right)$ has a system of generators $x_{1}, x_{2}, x_{3}, x_{i}, x_{8}, x_{9}, x_{11}, x_{15}$ of type $(M)$ [3]. Let $\pi: E_{6} \rightarrow \operatorname{Ad} E_{6}$ be the projection. The above generators can be chosen to satisfy

$$
\pi *\left(x_{i}\right)=x_{i} \quad \text { for } i=3,7,8,11,15 .
$$

Since coproducts commute with the projection, from the Prop. 3 we see that the coproducts $\psi^{*} x_{11}$ and $\psi^{*} x_{15}$ in $H^{*}\left(\operatorname{Ad} E_{0} ; Z_{3}\right)$ are not symmetric (by a definition of [5], p. 283). Then by [5], Prop. 2.5, we have the

Theorem 2. The Pontrjagin ring $H_{*}\left(\operatorname{Ad} E_{\hat{0}} ; Z_{\xi}\right)$ is not commutative.

§ 3. $\boldsymbol{g}^{*}: \boldsymbol{H}^{*}\left(\boldsymbol{E}_{7} ; \boldsymbol{Z}_{3}\right) \rightarrow \boldsymbol{H} *\left(\boldsymbol{E}_{\tilde{0}} ; \boldsymbol{Z}_{3}\right)$

1. By the Prop. II. 9 we see that $\Omega g^{*} \bmod 3$ is injective in deg. 10 . Hence $\Omega g^{*}\left(u_{10}\right) \neq 0$ and is transgressive in the fibration of loop space of $E_{0}$ by No. 1.3. Hence we can choose $u_{10}$ of $\Omega E_{7}$ such that

$$
\Omega g *\left(u_{10}\right)=u_{10}
$$

Then by the same discussion as No. 2.1 we can choose the generators $x_{11}$ and $x_{15}$ of $H^{*}\left(E_{7} ; Z_{3}\right)$ to satisfy

$$
g *\left(x_{11}\right)=x_{11}
$$




$$
x_{15}=\mathscr{P}^{1} x_{11} .
$$

Now $f^{*} g^{*} x_{15}=x_{15}$, and by $(4),(7),\left(7^{\prime}\right)$ and $\left(7^{\prime \prime}\right)$ we see that $f^{*} g^{*} \bmod 3$ is surjective. In another word

Proposition 4. $F_{4}$ is totally non homologous zero mod 3 in $E_{7} . f^{*} g^{*} \bmod$ 3 is bijective in deg. $\leqq 18$.

Then by Theorem WCSS the induced cohomology map of classifying spaces of $F_{4}$ and $E_{7}$ is bijective in deg. $\leqq 19$. Hence by the Cor. to the Prop. I. 2 we see the

Corollary. $H^{*}\left(B_{E_{7}} ; Z_{3}\right)=Z_{3}\left[y_{4}, y_{8}\right] \otimes \Lambda_{3}\left(y_{9}\right) /\left(y_{4} y_{9}, y_{8} y_{9}\right)$ in deg. $\leqq 19$.

2. The behaviors of generators $x_{11}, x_{15}$ of $H^{*}\left(E_{7} ; Z_{3}\right)$ in the universal spectral sequences and their coproducts are entirely the same as those of corresponding ones of $H^{*}\left(E_{\hat{0}} ; Z_{3}\right)$. So that we omit to write them down.

As a corollary of the Prop. 4 we see the following

Theorem 3. The Pontrjagin ring $H_{*}\left(E_{7} ; Z_{3}\right)$ in deg. $\leqq 18$, has 3-simple system of generators $v_{i}, i=3,7,8,11,15$, satisfying the relations described in Theorem I.2. $H_{*}\left(E_{7} ; Z_{3}\right)$ is not commutative.

Remark. The relations of non-commutativity $\left[v_{s}, v_{3}\right]=v_{11},\left[v_{s}, v_{7}\right]=v_{15}$ are inherited from those in $F_{4}$ and in $E_{6}$.

§ 4. $\boldsymbol{h}^{*}: \boldsymbol{H} *\left(\boldsymbol{E}_{8} ; \boldsymbol{Z}_{3}\right) \rightarrow \boldsymbol{H} *\left(\boldsymbol{E}_{7} ; \boldsymbol{Z}_{3}\right)$

1. By the Prop. II. 10 we see that $\Omega h^{*} \bmod 3$ is injective in deg. 14 . Then, in the same way with No. 3.1 we can choose the generators $u_{14} \in H^{*}\left(\Omega E_{8} ; Z_{3}\right)$ and $x_{15} \in H^{*}\left(E_{3} ; Z_{3}\right)$ to satisfy

$$
\Omega h^{*}\left(u_{14}\right)=u_{14}, \quad h^{*}\left(x_{15}\right)=x_{15} .
$$

Then, by similar discussions with those in preceding sections we have the following results.

PRoposition 5. The generator $x_{15}$ of $H^{*}\left(E_{8} ; Z_{3}\right)$ behave in the universal spectral sequence as follows:

$$
d_{i} \kappa_{i}^{2} x_{15}=0 \text { for } 2 \leqq i<9, d_{9} \kappa_{9}^{2} x_{15}=\kappa_{9}^{2}\left(y_{9} \otimes x_{7}\right) \neq 0 .
$$

The coproduct $\psi^{*} x_{15}$ in $H^{*}\left(E_{3} ; Z_{3}\right)$ is as follows:

$$
\psi^{*} x_{15}=1 \otimes x_{15}+x_{15} \otimes 1+x_{8} \otimes x_{7} .
$$


Corollary. $\quad H^{*}\left(B_{F_{8}} ; Z_{3}\right)=Z_{3}\left[y_{4}, y_{8}\right] \otimes \Lambda_{3}\left(y_{9}\right) /\left(y_{8} y_{9}\right)$ in deg. $\leqq 19$.

Theorem 4. The Pontrjagin ring $H_{*}\left(E_{5} ; Z_{3}\right)$ is not commutative and has non-zero elements $v_{3}, v_{7}, v_{8}, v_{15}$, which generates $H_{*}\left(E_{8} ; Z_{3}\right)$ in deg. $\leqq 18$ and satisfy the following relation of non-commutativity

$$
\left[v_{8}, v_{7}\right]=v_{15} \text {. }
$$

Remark. The relation $\left[v_{s}, v_{7}\right]=v_{15}$ is inherited from the corresponding one in $F_{4}, E_{6}$, and $E_{7}$.

\section{$\S 5$. On homotopy abelians}

Let $G \supset H$ be a group and a subgroup. The maps

$$
\lambda, \mu: H \times H \rightarrow G
$$

are defined by $\lambda(x, y)=x y=\mu(y, x)$ for $x, y \in H$. When $\lambda$ and $\mu$ are homotopic, then $H$ is called homotopy abelian in $G$, [19]. When $G=H$ and $G$ is homotopy abelian in $G$, then we say simply that $G$ is homotopy abelian.

As is well-known, if $G$ is homotopy-abelian, then the Pontrjagin products of $G$ over any coefficient field are commutative; if $H$ is homotopy abelian in $G$, then the Pontrjagin products of $H$ into $G$ over any coefficient field are commutative.

Hence, by Theorems 1, 2, 3 and 4 and the remarks to them we can see the following Theorems.

TheoRem 5. $E_{\hat{0}}, \operatorname{Ad} E_{6}, E_{7}, \operatorname{Ad} E_{\tilde{\tau}}$ and $E_{8}$ are not homotopy abelian.

Theorem 6. In the inclusions

$$
F_{4} \subset E_{0} \subset E_{7} \subset E_{3},
$$

every subgroup is not homotopy abelian in any group containing it.

Remark. Theorem 5, jointly with the results of James-Thomas [19], proves that every compact simple Lie group is not homotopy abelian.

The author knows another proof of Theorem 5 in the same line with the James-Thomas' proof [17] of homotopy non abelian of other compact simple Lie groups, cf. [20]. Nevertheless, I think the Theorem 6 is a new result.

\section{REFERENCES}

[1] S, Araki, On the homology of spinor groups, Mem. Fac. Sci., Kyusyu Univ., Ser. A, 9 (1955), 1-35. 
[2] S. Araki, Steenrod reduced powers in the spectral sequences associated with a fibering, Mem. Fac. Sci., Kyusyu Univ., Ser. A, 11 (1957), 15-64 and 81-97.

[3] S. Araki, A theorem on differential Hopf algebras and the cohomology mod 3 of the compact exceptional groups $E_{7}$ and $E_{8}$, to appear.

[4] A. Borel, Sur la cohomologie des espaces fibrés principaux et des espaces homogènes de groupes de Lie compacts, Ann. Math., 57 (1953), 115-207.

[5] A. Borel, Sur l'homologie et la cohomologie des groupes de Lie compacts connexes, Amer. J. Math., 76 (1954), 272-342.

[6] A. Borel, Sousgroupes commutatifs et torsion des groupes de Lie compacts, to appear.

[7] A. Borel and J. de Siebenthal, Les sous-groupes fermés de rang maximum des groupes de Lie clos, Comment. Math. Helvet., 23 (1949), 200-221.

[8] R. Bott, An application of the Morse theory to the topology of Lie groups, Bull. Soc. Math. France, 84 (1956), 251-281.

[9] R. Bott, The space of loops on a Lie group, Michigan Math. J., 5 (1958), 35-61.

[10] R. Bott and H. Samelson, Applications of the Morse theory to symmetric spaces, Amer. J. Math., 80 (1958), 964-1029.

[11] J. Kojima, On the Pontrjagin product mod 2 of spinor groups, Mem. Fac. Sci., Kyusyu Univ., Ser. A, 11 (1957), 1-14.

[12] T. Kudo, A transgression theorem, Mem. Fac. Sci., Kyusyu Univ., Ser. A, 9 (1956), 79-81.

[13] T. Kudo and S. Araki, Topology of $H_{n}$-spaces and $H$-squaring operations, Mem. Fac. Sci., Kyusyu Univ., Ser. A, 10 (1956), 85-120.

[14] H. Samelson, Beiträge zur Topologie der Gruppen-Mannigfaltigkeiten, Ann. Math., 42 (1941), 1091-1137.

[15] J.-P. Serre, Homologie singulière des espaces fibrés. Applications, Ann. Math., 54 (1951), 425-505.

[16] J. de Siebenthal, Sur les sous-groupes fermés connexes d'un groupes de Lie clos, Comment. Math. Helvet., 25 (1951), 210-256.

[17] E. Stiefel, Über eine Beziehung zwischen geschlossenen Lie'schen Gruppen und diskontinuierlichen Bewegungsgruppen..., Comment. Math. Helvet., 14 (1941), 350380 .

[18] A. S. Svarc, Dokl. Akad. Nauk. SSSR (N.S.), 104 (1955), 26-29.

[19] I. M. James and E. Thomas, Which Lie groups are homotopy abelian?, Proc. Nat. Acad. Sci., U.S.A., 45 (1959), 734-740.

[20] S. Araki, I. M. James and E. Thomas, Homotopy-abelian Lie groups, Bull. Amer. Math. Soc., 66 (1960), 324-326.

\section{The Institute for Advanced Study \\ and}

Kyusyu University, Japan 